\title{
Renormalized holographic subregion complexity under relevant perturbations
}

\author{
Dongmin Jang, ${ }^{a}$ Yoonbai Kim, ${ }^{a}$ O-Kab Kwon ${ }^{a}$ and D.D. Tolla ${ }^{a, b}$ \\ ${ }^{a}$ Department of Physics, BK21 Physics Research Division, \\ Autonomous Institute of Natural Science, Institute of Basic Science, Sungkyunkwan University, \\ Suwon 440-746, South Korea \\ ${ }^{b}$ University College, Sungkyunkwan University, \\ Suwon 440-746, South Korea \\ E-mail: dongmin@skku.edu, yoonbai@skku.edu, okab@skku.edu, \\ ddtolla@skku.edu
}

ABSTRACT: We construct renormalized holographic entanglement entropy (HEE) and subregion complexity (HSC) in the CV conjecture for asymptotically $\mathrm{AdS}_{4}$ and $\mathrm{AdS}_{5}$ geometries under relevant perturbations. Using the holographic renormalization method developed in the gauge/gravity duality, we obtain counter terms which are invariant under coordinate choices. We explicitly define different forms of renormalized HEE and HSC, according to conformal dimensions of relevant operators in the $d=3$ and $d=4$ dual field theories. We use a general embedding for arbitrary entangling subregions and showed that any choice of the coordinate system gives the same form of the counter terms, since they are written in terms of curvature invariants and scalar fields on the boundaries. We show an explicit example of our general procedure. Intriguingly, we find that a divergent term of the HSC in the asymptotically $\mathrm{AdS}_{5}$ geometry under relevant perturbations with operators of conformal dimensions in the range $0<\Delta<\frac{1}{2}$ and $\frac{7}{2}<\Delta<4$ cannot be cancelled out by adding any coordinate invariant counter term. This implies that the HSCs in these ranges of the conformal dimensions are not renormalizable covariantly. We also write the plot of the renormalization procedure in the case of asymptotically $\operatorname{AdS}_{d+1}$ geometries, with $d>4$.

KEYWORDS: Gauge-gravity correspondence, Renormalization Regularization and Renormalons

ARXIV EPRINT: 2001.10937 


\section{Contents}

1 Introduction 1

2 Renormalized HEE under relevant perturbations $\quad 4$

2.1 Renormalized HEE in asymptotically $\mathrm{AdS}_{4}$ geometry 4

2.2 Renormalized HEE in asymptotically $\mathrm{AdS}_{5}$ geometry 9

2.3 Comments on the case of asymptotically $\mathrm{AdS}_{d+1>5}$ geometry 12

3 Renormalized HSC under relevant perturbations $\quad \mathbf{1 5}$

3.1 Renormalized HSC in asymptotically $\mathrm{AdS}_{4}$ geometry 15

$\begin{array}{lll}3.2 & \text { Renormalized HSC in asymptotically } \mathrm{AdS}_{5} \text { geometry } & 18\end{array}$

3.3 Comments on the case of asymptotically $\mathrm{AdS}_{d+1>5}$ geometry 20

4 An example: the LLM geometry 21

4.1 The LLM geometry 21

4.2 Renormalized HEE and HSC in the mABJM theory 23

$\begin{array}{llr}5 & \text { Conclusion } & 27\end{array}$

$\begin{array}{ll}\text { A Renormalized HEE in pure } \mathrm{AdS}_{d+1} \text { geometry } & 29\end{array}$

$\begin{array}{ll}\text { B Renormalized HSC in pure } \operatorname{AdS}_{d+1} \text { geometry } & 31\end{array}$

\section{Introduction}

The holographic realizations of the entanglement entropy and the quantum complexity have established a connection between the gravity theory and quantum information theory through gauge/gravity duality [1-3]. The Ryu-Takanayagi (RT) conjecture [4-6] laid out a holographic way of calculating the entanglement entropy for a subregion $A$ in a $d$ dimensional field theory on the boundary of a $(d+1)$-dimensional bulk geometry of the dual gravity theory. According to the RT conjecture, the holographic entanglement entropy (HEE) is proportional to the area of $(d-1)$-dimensional bulk minimal hyper-surface $\Sigma_{A}$, which is homologous to the subspace $A$ in a $d$-dimensional constant time slice. The quantum complexity is also an important quantity in the information theory, which measures how many minimum simple gates to reach from a simple reference state to a target state. However, the notion is not well-defined in quantum field theory generally. There have been two proposals to calculate the quantum complexity in terms of the gauge/gravity, which are referred to as the CV (Complexity=Volume) conjecture [7] and the CA (Complexity=Action) conjecture $[8,9]$. These correspond to the complexity of a pure state in the whole boundary space of the dual quantum field theory. Natural generalizations of the 
$\mathrm{CV}$ and CA conjectures are holographic complexities of a mixed state for the reduced density matrix for a subregion $A$. These are known as the holographic subregion complexity (HSC) for the CV conjecture [10] or the CA conjecture [12]. Other studies on the HSC include [10-27]. In this paper, we construct renormalized HEE and HSC by focusing on the $\mathrm{CV}$ conjecture and hence the HSC in this paper refers to the quantity obtained through the $\mathrm{CV}$ conjecture.

The HSC states that the quantum complexity of a mixed state, which is produced by reducing the boundary state to a specific subregion $A$, is proportional to the volume of the extremal hyper-surface $\mathcal{B}_{A}$ enclosed by the boundary subregion $A$ and corresponding RT surface $\Sigma_{A}$. See figure 1. Therefore, in order to calculate the HSC, one has to fix the RT surface at first by solving equations of motion to minimize the codimension two hyper-surface ending on the boundary of the subregion $A$. Then the HSC is computed by

$$
\mathcal{C}_{A}=\frac{V\left(\mathcal{B}_{A}\right)}{8 \pi L G_{d+1}}
$$

where $L$ and $G_{d+1}$ are the AdS radius and the Newtonian constant in $(d+1)$-dimensions, respectively. It was also suggested that the quantity $\mathcal{C}_{A}$ in (1.1) can be interpreted as the fidelity susceptibility in the quantum information theory [10, 28].

The HEE and HSC involve integration over extremal subspaces that are extending to the asymptotic boundary. As a result, they are divergent due to the infinite area/volume of the extremal subspaces on the boundary. In the dual boundary field theory, these divergences correspond to the UV divergences, which are related to the short distance correlations, and it is necessary to renormalize those holographic quantities. One well-known method to renormalize the HEE is to cancel out the divergent terms by using differentiation with respect to a characteristic length scale of the entangling subregion [29]. See also [30]. However, this method depends on the shape of the entangling region and the choice of coordinate system. In order to overcome the disadvantages of the differentiation method, a systematic renormalization method known as the holographic renormalization [31-34] was applied to the renormalization of the HEE [35]. See also [36, 37]. Application of this method to the HSC for pure AdS spaces was also discussed in [12]. Renormalization of the holographic complexity for pure states in terms of the holographic renormalization method was studied in [38].

In this paper, we construct renormalized HEE and HSC with arbitrary entangling subregions for asymptotically $\mathrm{AdS}_{4}$ and $\mathrm{AdS}_{5}$ geometries ${ }^{1}$ under relevant perturbations by introducing a scalar field. We determine covariant counter terms on the cut-off boundary in terms of the holographic renormalization method. Our construction also can be applied to generic asymptotically AdS geometries, such as AdS black holes and AdS solitons, etc.

In the case of the HEE, recalling that the RT minimal hyper-surface $\Sigma_{A}$ is homologous to the subspace $A$, its boundary $\partial \Sigma_{A}$ is independent of the bulk stress tensor. However, the subleading divergences in the HEE are determined by the back reaction of the stress tensor

\footnotetext{
${ }^{1}$ We omit the case of the $\mathrm{AdS}_{3}$ geometry, which is similar to the case of the $\mathrm{AdS}_{5}$ geometry, however, we show the general procedure of how to cancel few leading divergences in the case of asymptotically $\operatorname{AdS}_{d+1}$ geometries, with $d>4$.
} 
on the geometry. In order to account for these subleading divergence, the counter terms should contain invariants of the matter fields in addition to the curvature invariants on $\partial \Sigma_{A}$. We determine the exact forms of these counter terms in asymptotically $\mathrm{AdS}_{4}$ and $\mathrm{AdS}_{5}$ geometries under relevant perturbations. The renormalized HEE with a disk entangling subregion in asymptotically $\mathrm{AdS}_{4}$ geometry under relevant perturbations was obtain in [35]. This result is also obtained from our result of the asymptotically $\mathrm{AdS}_{4}$ geometry.

In the case of the HSC, the curvature invariants on the cut-off boundary are dependent on the bulk stress tensor. Therefore, the counter terms to cancel the leading as well as subleading divergences can be just the integrals of the curvature invariants on the $(d-1)$ dimensional cut-off boundary. Like the HEE case, one can also include counter terms which explicitly contain invariants of the matter fields. However, they are redundant, because the nature of divergences obtained from those terms is the same as those obtained from the counter terms built from the curvature invariants on the $(d-1)$-dimensional cut-off boundary. Actually, this is expected due to the back reaction of the matter field deformations on the cut-off boundary geometry. Keeping this in mind, we express the counter terms explicitly in terms of the matter fields by replacing the contributions from the curvature invariants on the $(d-1)$-dimensional space by the appropriate matter field invariants. In addition, since the $(d-1)$-dimensional cut-off boundary meets the $(d-2)$-dimensional boundary $\partial \Sigma_{A}$ of the RT hyper-surface, there are always divergent terms, which are expressed in terms of integrals of curvature invariants on $\partial \Sigma_{A}$. We show that the complete counter terms for the HSC are expressed as integrals of the curvature invariants on the $(d-1)$-dimensional cut-off boundary plus integrals of the curvature invariants on the $(d-2)$-dimensional boundary of the RT hyper-surface. We apply this procedure to a particular example of an asymptotically $\mathrm{AdS}_{4}$ geometry obtained from the non-linear KK reduction of the 11-dimensional LLM geometry [39] and obtain coordinate independent finite results.

Intriguingly, we find that there exist a divergent term $\mathcal{O}\left(\epsilon^{\alpha-1}\right)$ with the range $0<\alpha<1$, which cannot be cancelled out by adding any curvature invariant, in the renormalization procedure of the HSC in the asymptotically $\mathrm{AdS}_{5}$ geometry under relevant perturbations. This implies that there is no renormalized HSC in the range $0<\alpha<1$, with this range of $\alpha$ corresponding to the conformal dimension of the relevant operators in the 4-dimensional dual field theory to be in the range $0<\Delta<\frac{1}{2}$ and $\frac{7}{2}<\Delta<4$. Here we also notice that the latter case does not violate the unitary bound $(\Delta \geq 1)$ for primary operators. It will be interesting if one figures out the physical reason of this phenomenon. To do that, one needs more investigations for other HSC, such as in the CA conjecture and other dimensions to resolve this problem.

The remaining parts of this paper are organized as follows. In section 2, we discuss the renormalization of $\mathrm{HEE}$ in an asymptomatically $\mathrm{AdS}_{4}$ and $\mathrm{AdS}_{5}$ geometries, which are obtained from the perturbation of the AdS geometries with a scalar field. We show the counter terms are determined by the curvature invariants on the boundary of the RT hypersurface as well as the scalar field. We comment on the generalization to higher dimensions as well. In section 3, we renormalize the HSC. We point out that the counter terms built just from the curvature invariants on the cut-off boundary are not enough to cancel the divergences and show the need for including the curvature invariants on the boundary of 
the RT hyper-surface. We obtain the forms of the counter terms in the asymptomatically $\mathrm{AdS}_{4}$ and $\mathrm{AdS}_{5}$ geometries. In the case of asymptomatically $\mathrm{AdS}_{d+1>5}$ geometries, we obtain the counter terms that are needed to cancel few leading divergences and summarize the complete renormalization procedure for the simple case of pure AdS geometry in the two appendices at the end. In section 4, we apply the general results of sections 2 and 3 to the KK reduction of the LLM geometry. We draw our conclusions in section 5 .

\section{Renormalized HEE under relevant perturbations}

The UV divergences in the EE and the quantum complexity naturally appear due to the strong entanglement near the boundary of entangling regions in quantum field theory. In the dual gravity theory, the corresponding HEE and HSC also have UV divergences, which should be renormalized before we associate physical phenomenon with the entanglement and the quantum complexity. In this section, we focus on the renormalization of the HEE in asymptotically $\mathrm{AdS}_{d+1}$ geometries, which are obtained by relevant perturbations that correspond to insertion of scalar fields in the dual gravity. A simple well-known way to renormalize the HEE is to use the differentiation for the HEE with respect to a characteristic length scale [29], for instance, the radius of the disk or the width of the strip of entangling regions. However, this method cannot be applicable for some entangling regions and depends on the choice of the spacetime coordinate. To overcome these drawbacks, a systematic way was proposed [35] by adopting the method of the holographic renormalization [31-34] in the gauge/gravity duality. For the relevant perturbation near the asymptotic region, the renormalized HEE for a disk in the asymptotically $\mathrm{AdS}_{4}$ geometry was obtained. Here we briefly review the method of ref. [35], and extend the method to some entangling regions for the asymptotically $\mathrm{AdS}_{4}$ and $\mathrm{AdS}_{5}$ geometries under relevant perturbations and also comment on higher dimensions.

In the next section, we consider the renoramalization of the HSC with arbitrary shapes of entangling regions on asymptotically $\mathrm{AdS}_{4}$ and $\mathrm{AdS}_{5}$ geometries. We propose new counter terms, which are genuine in the renormalization of the HSC.

\subsection{Renormalized HEE in asymptotically $\mathrm{AdS}_{4}$ geometry}

In the field theory, the relevant deformation of the $d$-dimensional CFT refers to inserting gauge invariant operators with conformal dimension $\Delta<d$, whereas in the dual gravity, this relevant perturbation is achieved by introducing a scalar field of mass $M^{2}=\Delta(\Delta-d)$ with $\Delta<d$. Therefore, for the holographic description of the EE under relevant perturbations in $d$-dimensional CFT, we start from the $(d+1)$-dimensional gravity action with negative cosmological constant coupled to a scalar field $\phi$,

$$
S_{g \phi}=\frac{1}{16 \pi G_{d+1}} \int d^{d+1} x \sqrt{-g}\left(\mathcal{R}-2 \Lambda-\frac{1}{2} \partial_{p} \phi \partial^{p} \phi-\frac{1}{2} M^{2} \phi^{2}-V(\phi)\right),
$$

where $G_{d+1}$ is the $(d+1)$-dimensional Newton's constant, $x^{p}=\left(z, x^{\mu}\right)$ with the $d$-dimensional boundary coordinates $x^{\mu}$, are the bulk coordinates with the holographic radial direction $z$, $\Lambda=-\frac{d(d-1)}{2 L^{2}}$ is the cosmological constant, and $V(\phi)$ denotes the potential with higher order 
self-couplings of the scalar field. Under the assumption of the Poincaré invariance for the coordinate $x^{\mu}$, the metric of an asymptotically $\mathrm{AdS}_{d+1}$ geometry in the Fefferman-Graham coordinate system is given by

$$
d s^{2}=g_{p q} d x^{p} d x^{q}=\frac{L^{2}}{z^{2}}\left(d z^{2}+(1+h(z)) \eta_{\mu \nu} d x^{\mu} d x^{\nu}\right) .
$$

The metric fluctuation $h(z)$, which vanishes at the boundary $(z=0)$, measures the deviation from the pure $\mathrm{AdS}_{d+1}$ geometry, due to the nonvanishing contribution of the Poincaré invariant scalar field $\phi=\phi(z)$.

Plugging (2.2) and the scalar field ansatz $\phi=\phi(z)$ into the Einstein equation and the equation of motion for the scalar field in 4-dimensions, we obtain

$$
\begin{array}{r}
2 z(1+h) h^{\prime \prime}-2 z h^{2}+2(1+h) h^{\prime}+z(1+h)^{2} \phi^{2}=0, \\
z^{2}(1+h) \phi^{\prime \prime}-2 z(1+h) \phi^{\prime}+\frac{3}{2} z^{2} h^{\prime} \phi^{\prime}-L^{2} M^{2}(h+1) \phi+\cdots=0,
\end{array}
$$

where the ellipses denote contributions from the potential $V(\phi)$. In the asymptotic region $(z \rightarrow 0)$, there are two independent solutions of the equations in (2.3),

$$
\begin{array}{ll}
\phi_{a}(z)=s_{0} z^{3-\Delta}+s_{1} z^{3(3-\Delta)}+\cdots & \Longrightarrow h_{a}(z)=-\frac{s_{0}^{2}}{8} z^{2(3-\Delta)}+h_{1} z^{4(3-\Delta)}+\cdots, \\
\phi_{b}(z)=v_{0} z^{\Delta}+v_{1} z^{3 \Delta}+\cdots & \Longrightarrow
\end{array}
$$

where $\Delta=\frac{1}{2}\left(3+\sqrt{9+4 M^{2} L^{2}}\right)$ is the conformal dimension of a gauge invariant operator dual to the scalar field $\phi$. The operator is relevant for $\Delta<3$. According to the GKP-W relation $[2,3]$ in the gauge/gravity duality, we have the relations

$$
s_{0} \sim J_{\mathcal{O}^{\Delta}}, \quad v_{0} \sim\left\langle\mathcal{O}^{\Delta}\right\rangle,
$$

where $J_{\mathcal{O}^{\Delta}}$ and $\left\langle\mathcal{O}^{\Delta}\right\rangle$ are the source and the vacuum expectation value of a gauge invariant operator $\mathcal{O}^{\Delta}$ with conformal dimension $\Delta$, respectively. The coefficients $\left(s_{1}, h_{1}, \cdots\right)$ in $(2.4)$ are determined in terms of $s_{0}$ and $\left(v_{1}, \tilde{h}_{1}, \cdots\right)$ are determined in terms of $v_{0}$, by inserting these solutions into (2.3) and solving order by order in $z$. We will show that, the way these solutions depend on the holographic coordinate $z$, determines the forms of the divergent terms in the HEE and HSC as well as the forms of the appropriate counter terms.

The RT conjecture states that the HEE of a subregion $A$ and its complement $A^{c}$, which lies on the boundary of $(d+1)$-dimensional bulk geometry, is given by

$$
S_{A}=\frac{\operatorname{Min}\left(\mathcal{A}_{\Sigma_{A}}\right)}{4 G_{d+1}}=\frac{1}{4 G_{d+1}} \int d z \int d^{d-2} \sigma^{a} \sqrt{\gamma}
$$

where $\operatorname{Min}\left(\mathcal{A}_{\Sigma_{A}}\right)$ denotes the minimal area of a bulk static hyper-surface $\Sigma_{A}$, which has the same boundary with the subregion $A$. The induced metric on $\Sigma_{A}$ with the target space metric $g_{p q}$ in (2.2) is defined as $\gamma_{\alpha \beta}=\frac{\partial x^{p}}{\partial \sigma^{\alpha}} \frac{\partial x^{q}}{\partial \sigma^{\beta}} g_{p q}$ with the worldvolume coordinate $\sigma^{\alpha}=\left\{z, \sigma^{a}\right\}, a=1, \cdots, d-2$. In [35], the authors introduced a parametrization of the 
embedding of the static surface $\Sigma_{A}$ at a constant time $t=t_{0}$ with arbitrary entangling region into the bulk space by setting

$$
x^{a}=\sigma^{a} \quad \text { and } \quad x^{d-1} \equiv y=w\left(z, x^{a}\right) .
$$

It seems that the embedding $(2.7)$ with $(d-1)$-dimensional independent parameters can express most shapes of entangling subregions, since the resulting hyper-surfaces $\Sigma_{A}$ 's are also $(d-1)$-dimensional geometries. In this sense, the embedding (2.7) is applicable to arbitrary shapes of entangling subregions.

For $d=3$, this embedding is written as

$$
x^{p}=\left(t=t_{0}, z, x, y=w(z, x)\right) .
$$

The induced metric on the surface $\Sigma_{A}$ is then given by

$$
\gamma_{z z}=\frac{L^{2}}{z^{2}}\left(1+w^{\prime 2}(1+h)\right), \quad \gamma_{z x}=\frac{L^{2}}{z^{2}} \dot{w} w^{\prime}(1+h), \quad \gamma_{x x}=\frac{L^{2}}{z^{2}}(1+h)\left(1+\dot{w}^{2}\right),
$$

where $\sigma^{\alpha}=(z, x)$ are coordinates on the surface, and we have used the notations $w^{\prime}=\partial_{z} w$, $\dot{w}=\partial_{x} w$. Then the area of the surface $\Sigma_{A}$ is calculated as

$$
\mathcal{A}_{\Sigma_{A}}=\int d x \int d z \sqrt{\operatorname{det} \gamma_{\alpha \beta}}=L^{2} \int d x \int d z \frac{(1+h)^{1 / 2}}{z^{2}} \sqrt{1+\dot{w}^{2}+(1+h) w^{2}} .
$$

The following Euler-Lagrange equation derived from this action determines the minimal area surface:

$$
\begin{aligned}
& z(1+h) w^{\prime \prime}+z \ddot{w}-2(1+h) w^{\prime}+\frac{3}{2} z h^{\prime} w^{\prime} \\
& -\frac{z}{1+\dot{w}^{2}+(1+h) w^{\prime 2}}\left(\dot{w}^{2} \ddot{w}+\frac{1}{2}(1+h) h^{\prime} w^{\prime 3}+2(1+h) \dot{w} w^{\prime} \dot{w}^{\prime}+(1+h)^{2} w^{\prime 2} w^{\prime \prime}\right)=0 .
\end{aligned}
$$

Near the asymptotic limit $z \rightarrow 0$, the equation (2.11) is solved order by order in $z$ by inserting the expansion

$$
\begin{aligned}
h(z) & =h_{0} z^{\alpha}+h_{1} z^{2 \alpha}+\cdots, \\
w(x, z) & =w_{0}(x)+w_{2}(x) z^{2}+w_{\alpha+2}(x) z^{\alpha+2}+w_{4}(x) z^{4}+\cdots,
\end{aligned}
$$

where we read from (2.4) that $\alpha>0$ and $\alpha$ is the smaller of $2(3-\Delta)$ and $2 \Delta$. From the leading order of (2.11), one can determines $w_{2}$ in terms of $w_{0}$ as

$$
w_{2}=\frac{\ddot{w}_{0}}{2\left(1+\dot{w}_{0}^{2}\right)} .
$$

Similarly, the higher order coefficients can also be determined, however, those are not required to obtain the gauge invariant counter term in the asymptotically $\mathrm{AdS}_{4}$ geometry. Plugging the expansion (2.12) into (2.10) and introducing a cut-off $z=\epsilon$ shown in figure 1, we obtain the regularized $\mathrm{HEE}$,

$$
S_{A}^{\mathrm{reg}}=\frac{L^{2}}{4 G_{4}} \int d x \int_{\epsilon}^{z_{m}} d z \frac{(1+h)^{1 / 2}}{z^{2}} \sqrt{1+\dot{w}_{0}^{2}}\left(1+\frac{2 w_{2}^{2}+\dot{w}_{0} \dot{w}_{2}}{1+\dot{w}_{0}^{2}} z^{2}+\mathcal{O}\left(z^{\alpha+2}, z^{4}\right)\right),
$$




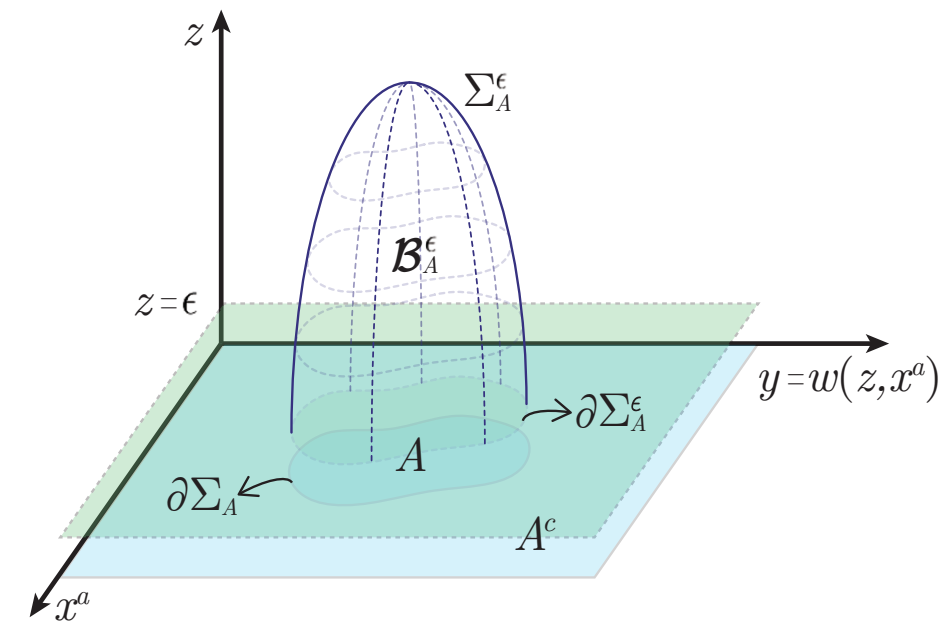

Figure 1. The RT surface and the subregion volume embedded into the asymptotically $\mathrm{AdS}_{d+1}$ geometry.

where $z_{m}$ denotes the maximum value of $z$ and is determined from the boundary condition $w^{\prime}\left(z_{m}, x\right) \rightarrow \infty$. Using the expansion of $h(z)$ in (2.12), one can rewrite the regularized HEE in (2.14) as

$$
S_{A}^{\mathrm{reg}}=\frac{L^{2}}{4 G_{4}} \int d x \int_{\epsilon}^{z_{m}} d z \sqrt{1+\dot{w}_{0}^{2}}\left(z^{-2}+\frac{h_{0}}{2} z^{\alpha-2}+\mathcal{O}\left(z^{2 \alpha-2}\right)\right) .
$$

Evaluating the $z$ integral, we obtain

$$
S_{A}^{\mathrm{reg}}=\left\{\begin{array}{lr}
\frac{L^{2}}{4 G_{4}} \int d x \sqrt{1+\dot{w}_{0}^{2}}\left(\frac{1}{\epsilon}-\frac{h_{0} \epsilon^{\alpha-1}}{2(\alpha-1)}+\mathcal{O}\left(\epsilon^{2 \alpha-1}\right)+\cdots\right), & 0<\alpha<1 \\
\frac{L^{2}}{4 G_{4}} \int d x \sqrt{1+\dot{w}_{0}^{2}}\left(\frac{1}{\epsilon}-\frac{h_{0}}{2} \ln \left(\frac{\epsilon}{\ell}\right)+\cdots\right), & \alpha=1, \\
\frac{L^{2}}{4 G_{4}} \int d x \sqrt{1+\dot{w}_{0}^{2}}\left(\frac{1}{\epsilon}+\cdots\right), & \alpha>1
\end{array}\right.
$$

where for the $\alpha=1$ case, we have introduced an arbitrary length scale $\ell$, which implies that the renormalized HEE will be renormalization scheme dependent. From now onwards the ellipses denote less divergent terms and finite terms. The ranges of $\alpha$ in the above equation refers to the following ranges of the conformal dimension $\Delta$,

$$
\begin{aligned}
0<\alpha<1 & \Longleftrightarrow 0<\Delta<\frac{1}{2} \quad \text { or } \quad \frac{5}{2}<\Delta<3, \\
\alpha=1 & \Longleftrightarrow \Delta=\frac{1}{2}, \frac{5}{2}, \\
\alpha>1 & \Longleftrightarrow \frac{1}{2}<\Delta<\frac{5}{2} .
\end{aligned}
$$

Based on the value of the conformal dimension $\Delta$, the value of $h_{0}$ in (2.16) is determined, for instance, $h_{0}=-\frac{s_{0}^{2}}{8} \sim J_{\mathcal{O}^{\Delta}}^{2}$ for $\frac{3}{2}<\Delta<3$ and $h_{0}=-\frac{v_{0}^{2}}{8} \sim\left\langle\mathcal{O}^{\Delta}\right\rangle^{2}$ for $0<\Delta<\frac{3}{2}$, where we have used the GKP-W relations in (2.5). 
In general, the divergences in HEE are cancelled by counter terms which are composed of the invariant quantities built by the scalar field $\phi$, the induced metric $\tilde{\gamma}_{a b}$, and the extrinsic curvature $\tilde{K}_{a b}$ on the boundary $\partial \Sigma_{A}^{\epsilon}$ of the regularized minimal surface $\Sigma_{A}^{\epsilon}$ [35]. See figure 1. In our case, the boundary $\partial \Sigma_{A}^{\epsilon}$ is a one dimensional curve and its embedding into the cut-off surface $z=\epsilon$ is parametrized as $x^{i}=(x, y=w(\epsilon, x))$. The induced metric and the extrinsic curvature are given by

$$
\begin{aligned}
\tilde{\gamma}_{x x} & =\frac{L^{2}(1+h)}{\epsilon^{2}}\left(1+\dot{w}^{2}\right), & \tilde{K}_{x x} & =\frac{L(1+h)^{\frac{1}{2}} \ddot{w}}{\epsilon\left(1+\dot{w}^{2}\right)^{5 / 2}}, \\
\tilde{K}_{x y} & =\frac{L(1+h)^{\frac{1}{2}} \ddot{w} \dot{w}}{\epsilon\left(1+\dot{w}^{2}\right)^{5 / 2}}, & \tilde{K}_{y y} & =\frac{L(1+h)^{\frac{1}{2}} \ddot{w} \dot{w}^{2}}{\epsilon\left(1+\dot{w}^{2}\right)^{5 / 2}} .
\end{aligned}
$$

The trace of the extrinsic curvature is

$$
\tilde{K}=g^{i j} \tilde{K}_{i j}=\frac{\epsilon}{L \sqrt{1+h}}\left(\frac{\ddot{w}_{0}}{\left(1+\dot{w}_{0}^{2}\right)^{3 / 2}}+\mathcal{O}\left(\epsilon^{2}\right)\right)=\frac{\epsilon}{L \sqrt{1+h}}\left(\frac{2 w_{2}}{\left(1+\dot{w}_{0}^{2}\right)^{1 / 2}}+\mathcal{O}\left(\epsilon^{2}\right)\right),
$$

where we have used the result in (2.13) in the last step. We notice that non of the divergences in (2.16) are related to $\tilde{K}$. Actually, such extrinsic curvature plays a role in the renormalized HEE if one consider the $d>3$ cases. See $d=4$ case in the next subsection. Therefore, in the case at hand the counter terms contain only the invariants of $\phi$ and $\tilde{\gamma}_{a b}$.

The counter term required to cancel the leading divergence for $\alpha>0$ in (2.16) is given by

$$
S_{\mathrm{ct}}^{(1)}=-\frac{L}{4 G_{4}} \int d x \sqrt{\tilde{\gamma}}=-\frac{L^{2}}{4 G_{4}} \int d x \sqrt{1+\dot{w}_{0}^{2}}\left(\frac{1}{\epsilon}+\frac{h_{0}}{2} \epsilon^{\alpha-1}+\mathcal{O}\left(\epsilon^{2 \alpha-1}\right)\right) .
$$

Adding this counter term to (2.16), we obtain

$$
S_{A}^{\mathrm{reg}}+S_{\mathrm{ct}}^{(1)}=\left\{\begin{array}{lr}
\frac{L^{2}}{4 G_{4}} \int d x \sqrt{1+\dot{w}_{0}^{2}}\left(-\frac{\alpha h_{0} \epsilon^{\alpha-1}}{2(\alpha-1)}+\mathcal{O}\left(\epsilon^{2 \alpha-1}\right)+\cdots\right), & 0<\alpha<1 \\
\frac{L^{2}}{4 G_{4}} \int d x \sqrt{1+\dot{w}_{0}^{2}}\left(-\frac{h_{0}}{2} \ln \left(\frac{\epsilon}{\ell}\right)+\cdots\right), & \alpha=1 . \\
\frac{L^{2}}{4 G_{4}} \int d x \sqrt{1+\dot{w}_{0}^{2}}(\text { finite terms }), & \alpha>1
\end{array}\right.
$$

From the solutions in (2.4) we note

$$
\phi^{2}=\phi_{0}^{2} z^{\alpha}+\cdots=-8 h_{0} z^{\alpha}+\cdots,
$$

where $\phi_{0}=s_{0}$ for the first solution and $\phi=v_{0}$ for the second solution in (2.4). Therefore, the counter terms that cancel the subleading divergences in (2.21) are given by

$$
S_{\mathrm{ct}}^{(2)}=\left\{\begin{array}{lc}
-\frac{\alpha L}{64(\alpha-1) G_{4}} \int d x \sqrt{\tilde{\gamma}} \phi^{2}=\frac{L^{2}}{4 G_{4}} \int d x \sqrt{1+\dot{w}_{0}^{2}}\left(\frac{\alpha h_{0} \epsilon^{\alpha-1}}{2(\alpha-1)}+\cdots\right), & 0<\alpha<1 \\
-\frac{L}{64 G_{4}} \ln \left(\frac{\epsilon}{\ell}\right) \int d x \sqrt{\tilde{\gamma}} \phi^{2}=\frac{L^{2}}{4 G_{4}} \int d x \sqrt{1+\dot{w}_{0}^{2}}\left(\frac{h_{0}}{2} \ln \left(\frac{\epsilon}{\ell}\right)+\cdots\right), & \alpha=1
\end{array},\right.
$$


where we notice that the renormalized HEE for $\alpha>1$ was already obtained in (2.21). For $0<\alpha<1$, there is still remaining divergence of the order $\epsilon^{2 \alpha-1}$. This divergence and other less divergent terms $\mathcal{O}\left(\epsilon^{n \alpha-1}\right)$ are cancelled by the counter terms built from higher powers of $\tilde{K}$ and $\phi$. From (2.21) and (2.23), we define a coordinate independent renormalized HEE of entangling regions with the embedding (2.7) under relevant perturbations

$$
S_{A}^{\mathrm{ren}}=S_{A}^{\mathrm{reg}}+S_{\mathrm{ct}}^{(1)}+S_{\mathrm{ct}}^{(2)} .
$$

These renormalized HEEs under relevant perturbations reproduce those for the disk entangling region in [35].

\subsection{Renormalized HEE in asymptotically $\mathrm{AdS}_{5}$ geometry}

In order to test the generality of the renormalization procedure we discussed in the previous subsection, lets extend it to the case of an asymptotically $\mathrm{AdS}_{5}$ geometry under relevant perturbations. Similarly with the case of the asymptotically $\mathrm{AdS}_{4}$ geometry, we start from the action (2.1). Under assumption of the Poincare invariance for the coordinate $x^{\mu}$, the asymptotically $\mathrm{AdS}_{5}$ metric in the Fefferman-Graham coordinate system is given by

$$
d s^{2}=\frac{L^{2}}{z^{2}}\left(d z^{2}+(1+h(z)) \eta_{\mu \nu} d x^{\mu} d x^{\nu}\right) \quad \text { with } x^{\mu}=\left(t, x_{1}, x_{2}, y\right) .
$$

Then one can write the two independent solutions to equations of motions for the metric fluctuation and the corresponding scalar field as

$$
\begin{aligned}
& \phi_{a}(z)=s_{0} z^{4-\Delta}+s_{1} z^{3(4-\Delta)}+\cdots \quad \Longrightarrow \quad h_{a}(z)=-\frac{s_{0}^{2}}{12} z^{2(4-\Delta)}+h_{1} z^{4(4-\Delta)}+\cdots, \\
& \phi_{b}(z)=v_{0} z^{\Delta}+v_{1} z^{3 \Delta}+\cdots \quad \Longrightarrow \quad h_{b}(z)=-\frac{v_{0}^{2}}{12} z^{2 \Delta}+\tilde{h}_{1} z^{4 \Delta}+\cdots .
\end{aligned}
$$

The RT minimal area of the hyper-surface $\Sigma_{A}$ with entangling regions denoted in (2.7) for the asymptotically $\mathrm{AdS}_{5}$ geometry is a three dimensional manifold parametrized by the embedding

$$
x^{p}=\left(z, t=t_{0}, x_{1}, x_{2}, y=w\left(z, x_{1}, x_{2}\right)\right) .
$$

See figure 1. The induce metric on $\Sigma_{A}$ is given by

$$
\begin{aligned}
& \gamma_{z z}=\frac{L^{2}}{z^{2}}\left(1+w^{\prime 2}(1+h)\right), \quad \gamma_{z a}=\frac{L^{2}}{z^{2}} \partial_{a} w w^{\prime}(1+h), \\
& \gamma_{a b}=\frac{L^{2}}{z^{2}}(1+h)\left(\delta_{a b}+\partial_{a} w \partial_{b} w\right),
\end{aligned}
$$

where $\partial_{a}=\left(\partial_{x^{1}}, \partial_{x^{2}}\right), w^{\prime}=\partial_{z} w$. Then the HEE is determined by the minimum value of the area of this hyper-surface given by

$$
\mathcal{A}_{\Sigma_{A}}=L^{3} \int d^{2} x \int d z \frac{(1+h)}{z^{3}} \sqrt{1+\left(\partial_{a} w\right)^{2}+(1+h) w^{\prime 2}}
$$


The minimum area equation derived from this action is read as

$$
\begin{aligned}
& z(1+h) w^{\prime \prime}+z \partial_{a}^{2} w-3(1+h) w^{\prime}+2 z h^{\prime} w^{\prime} \\
& -\frac{z}{1+\partial_{c} w^{2}+(1+h) w^{\prime 2}}\left[\partial_{a} w \partial_{b} w \partial_{a} \partial_{b} w+(1+h)^{2}\left(w^{\prime 2} w^{\prime \prime}+\frac{\frac{1}{2} h^{\prime} w^{\prime 3}+2 w^{\prime} \partial_{a} w \partial_{a} w^{\prime}}{1+h}\right)\right]=0 .
\end{aligned}
$$

We introduce the asymptotic expansion of $w\left(z, x_{1}, x_{2}\right)$ and solve this equation order by order in $z$. In this case the iteration breaks down at $z^{d=4}$ order, and one needs to introduce logarithmic term at this order. We can also read the asymptotic expansion of $h(z)$ from (2.26),

$$
\begin{aligned}
h(z) & =h_{0} z^{\alpha}+h_{1} z^{2 \alpha}+\cdots, \\
w\left(z, x^{a}\right) & =\left\{\begin{array}{lr}
w_{0}\left(x^{a}\right)+w_{2}\left(x^{a}\right) z^{2}+w_{\alpha+2}\left(x^{a}\right) z^{\alpha+2}+\cdots, & 0<\alpha<2 \\
w_{0}\left(x^{a}\right)+w_{2}\left(x^{a}\right) z^{2}+w_{4}\left(x^{a}\right) z^{4}+\tilde{w}_{4}\left(x^{a}\right) z^{4} \ln (z)+\cdots, & \alpha \geq 2
\end{array},\right.
\end{aligned}
$$

where $\alpha$ is the smaller of $2(4-\Delta)$ and $2 \Delta$, and then $\alpha$ and $\Delta$ have the relations

$$
\begin{aligned}
0<\alpha<2 & \Longleftrightarrow 0<\Delta<1 \quad \text { or } \quad 3<\Delta<4, \\
\alpha=2 & \Longleftrightarrow \Delta=1,3, \\
\alpha>2 & \Longleftrightarrow 1<\Delta<3 .
\end{aligned}
$$

The $w_{n}$ 's in (2.31) are determined in terms of $w_{0}$ by solving the minimal area equation (2.30). For our purpose in this and the next subsections, we only need $w_{2}$ and $w_{\alpha+2}(0<\alpha<2)$. They are expressed as

$$
w_{2}=\frac{1}{4}\left(\partial_{a}^{2} w_{0}-\frac{\partial_{a} w_{0} \partial_{b} w_{0} \partial_{a} \partial_{b} w_{0}}{1+\left(\partial_{c} w_{0}\right)^{2}}\right), \quad w_{\alpha+2}=-\frac{4 h_{0}(\alpha-1) w_{2}}{\alpha^{2}-4} .
$$

Plugging the expansion (2.31) into (2.29) and introducing the cut-off surface $z=\epsilon$, the HEE is given by

$$
S_{A}^{\mathrm{reg}}=\frac{L^{3}}{4 G_{5}} \int d^{2} x \int_{\epsilon}^{z_{m}} d z \frac{(1+h) \sqrt{1+\left(\partial_{c} w_{0}\right)^{2}}}{z^{3}}\left[1+\frac{2 w_{2}^{2}+\partial_{a} w_{0} \partial_{a} w_{2}}{1+\left(\partial_{c} w_{0}\right)^{2}} z^{2}+\mathcal{O}\left(z^{\alpha+2}\right)\right] .
$$

Integrating by parts the second term in the square bracket in (2.34) and then using the result in (2.33), we obtain

$$
S_{A}^{\mathrm{reg}}=\frac{L^{3}}{4 G_{5}} \int d^{2} x \int_{\epsilon}^{z_{m}} d z \sqrt{1+\left(\partial_{c} w_{0}\right)^{2}}\left[\frac{1}{z^{3}}-\frac{2 w_{2}^{2}}{1+\left(\partial_{c} w_{0}\right)^{2}} \frac{1}{z}+h_{0} z^{\alpha-3}+\mathcal{O}\left(z^{\alpha-1}\right)\right],
$$

where $h_{0}=-\frac{s_{0}^{2}}{12} \sim J_{\mathcal{O}^{\Delta}}^{2}$ for $2<\Delta<4$ and $h_{0}=-\frac{v_{0}^{2}}{12} \sim\left\langle\mathcal{O}^{\Delta}\right\rangle^{2}$ for $0<\Delta<2$, and $w_{2}$ is expressed in terms of $w_{0}$ in (2.33). We carry out the $z$ integration and obtain

$$
S_{A}^{\mathrm{reg}}=\left\{\begin{array}{lc}
\frac{L^{3}}{4 G_{5}} \int d^{2} x \sqrt{1+\left(\partial_{c} w_{0}\right)^{2}}\left[\frac{1}{2 \epsilon^{2}}+\frac{2 w_{2}^{2}}{1+\left(\partial_{c} w_{0}\right)^{2}} \ln \left(\frac{\epsilon}{\ell}\right)-\frac{h_{0} \epsilon^{\alpha-2}}{\alpha-2}+\cdots\right], & 0<\alpha<2 \\
\frac{L^{3}}{4 G_{5}} \int d^{2} x \sqrt{1+\left(\partial_{c} w_{0}\right)^{2}}\left[\frac{1}{2 \epsilon^{2}}+\frac{2 w_{2}^{2}}{1+\left(\partial_{c} w_{0}\right)^{2}} \ln \left(\frac{\epsilon}{\ell}\right)-h_{0} \ln \left(\frac{\epsilon}{\ell}\right)+\cdots\right], & \alpha=2, \\
\frac{L^{3}}{4 G_{5}} \int d^{2} x \sqrt{1+\left(\partial_{c} w_{0}\right)^{2}}\left[\frac{1}{2 \epsilon^{2}}+\frac{2 w_{2}^{2}}{1+\left(\partial_{c} w_{0}\right)^{2}} \ln \left(\frac{\epsilon}{\ell}\right)+\cdots\right], & \alpha>2
\end{array}\right.
$$


where we also introduce some length scale $\ell$ like the case of the asymptotically $\mathrm{AdS}_{4}$ in the previous subsection. Next we fix the counter terms which are composed of the invariant quantities of the scalar field $\phi$, the induced metric, and extrinsic curvature on the boundary space $\partial \Sigma_{A}^{\epsilon}$. In this case the boundary space is a 2-dimensional surface embedded in the cut-off surface $z=\epsilon$ as $x^{i}=\left(x_{1}, x_{2}, y=w\left(\epsilon, x_{1}, x_{2}\right)\right)$. The induced metric and the extrinsic curvature are obtained as

$$
\begin{aligned}
\tilde{\gamma}_{a b} & =\frac{L^{2}}{\epsilon^{2}}(1+h)\left(\delta_{a b}+\partial_{a} w \partial_{b} w\right), \\
\tilde{K}_{a b} & =\frac{L(1+h)^{1 / 2}}{\epsilon \sqrt{1+\left(\partial_{c} w\right)^{2}}}\left(\partial_{a} \partial_{b} w-\frac{\partial_{a} w \partial_{b} \partial_{d} w \partial_{d} w+\partial_{b} w \partial_{a} \partial_{d} w \partial_{d} w}{1+\left(\partial_{c} w\right)^{2}}+\frac{\partial_{a} w \partial_{b} w \partial_{d} w \partial_{e} w \partial_{d} \partial_{e} w}{\left(1+\left(\partial_{c} w\right)^{2}\right)^{2}}\right), \\
\tilde{K}_{a y} & =\frac{L(1+h)^{1 / 2}}{\epsilon \sqrt{1+\left(\partial_{c} w\right)^{2}}}\left(\frac{\partial_{a} \partial_{b} \partial_{b} w}{1+\left(\partial_{c} w\right)^{2}}-\frac{\partial_{a} w \partial_{b} w \partial_{d} w \partial_{b} \partial_{d} w}{\left(1+\left(\partial_{c} w\right)^{2}\right)^{2}}\right), \\
\tilde{K}_{y y} & =\frac{L(1+h)^{1 / 2}}{\epsilon \sqrt{1+\left(\partial_{c} w\right)^{2}}}\left(\frac{\partial_{a} w \partial_{b} w \partial_{a} \partial_{b} w}{\left(1+\left(\partial_{c} w\right)^{2}\right)^{2}}\right) .
\end{aligned}
$$

Using the asymptotic expansion in (2.31), the trace of the extrinsic curvature is given by

$$
\tilde{K}=\frac{\epsilon(1+h)^{-1 / 2}}{L \sqrt{1+\left(\partial_{c} w_{0}\right)^{2}}}\left(\partial_{a}^{2} w_{0}-\frac{\partial_{a} w_{0} \partial_{b} w_{0} \partial_{a} \partial_{b} w_{0}}{1+\left(\partial_{c} w_{0}\right)^{2}}\right)+\mathcal{O}\left(\epsilon^{3}\right)=\frac{4 \epsilon(1+h)^{-1 / 2}}{L \sqrt{1+\left(\partial_{c} w_{0}\right)^{2}}} w_{2}+\mathcal{O}\left(\epsilon^{3}\right),
$$

where in the last step we have used the expression of $w_{2}$ in (2.33).

The first counter term that cancels the leading order divergence in (2.36) is

$$
S_{\mathrm{ct}}^{(1)}=-\frac{L}{8 G_{5}} \int d^{2} x \sqrt{\tilde{\gamma}}=-\frac{L^{3}}{4 G_{5}} \int d^{2} x \sqrt{1+\left(\partial_{c} w_{0}\right)^{2}}\left[\frac{1}{2 \epsilon^{2}}+\frac{h_{0}}{2} \epsilon^{\alpha-2}+\cdots\right] .
$$

Adding the counter term (2.39) to (2.36), we obtain

$$
S_{A}^{\mathrm{reg}}+S_{\mathrm{ct}}^{(1)}=\left\{\begin{array}{lr}
\frac{L^{3}}{4 G_{5}} \int d^{2} x \sqrt{1+\left(\partial_{c} w_{0}\right)^{2}}\left[\frac{2 w_{2}^{2}}{1+\left(\partial_{c} w_{0}\right)^{2}} \ln \left(\frac{\epsilon}{\ell}\right)-\frac{\alpha h_{0} \epsilon^{\alpha-2}}{2(\alpha-2)}+\cdots\right], & 0<\alpha<2 \\
\frac{L^{3}}{4 G_{5}} \int d^{2} x \sqrt{1+\left(\partial_{c} w_{0}\right)^{2}}\left[\frac{2 w_{2}^{2}}{1+\left(\partial_{c} w_{0}\right)^{2}} \ln \left(\frac{\epsilon}{\ell}\right)-h_{0} \ln \left(\frac{\epsilon}{\ell}\right)+\cdots\right], & \alpha=2 . \\
\frac{L^{3}}{4 G_{5}} \int d^{2} x \sqrt{1+\left(\partial_{c} w_{0}\right)^{2}}\left[\frac{2 w_{2}^{2}}{1+\left(\partial_{c} w_{0}\right)^{2}} \ln \left(\frac{\epsilon}{\ell}\right)+\cdots\right], & \alpha>2
\end{array}\right.
$$

The universal logarithmic divergences [40] which are present in the all the three ranges of $\alpha$ are cancelled by the counter term composed of trace of the extrinsic curvature in (2.38). As we mentioned in the previous subsection this term is absent for $d<4$. The required counter term is ${ }^{2}$

$$
S_{\mathrm{ct}}^{(2)}=-\frac{L^{3}}{32 G_{5}} \ln \left(\frac{\epsilon}{\ell}\right) \int d^{2} x \sqrt{\tilde{\gamma}} \tilde{K}^{2}=-\frac{L^{3}}{4 G_{5}} \ln \left(\frac{\epsilon}{\ell}\right) \int d^{2} x\left[\frac{2 w_{2}^{2}}{\sqrt{1+\left(\partial_{c} w_{0}\right)^{2}}}+\mathcal{O}\left(\epsilon^{2}\right)+\cdots\right] .
$$

\footnotetext{
${ }^{2}$ In [35], it was argued that odd powers of $\tilde{K}$ can not enter the counter terms because the renormalized HEE for the subspace $A$ and its complement $A^{c}$, which has opposite sign of the extrinsic curvature, must be the same.
} 
Then we obtain

$$
S_{A}^{\mathrm{reg}}+S_{\mathrm{ct}}^{(1)}+S_{\mathrm{ct}}^{(2)}=\left\{\begin{array}{cc}
\frac{L^{3}}{4 G_{5}} \int d^{2} x \sqrt{1+\left(\partial_{c} w_{0}\right)^{2}}\left[-\frac{\alpha h_{0} \epsilon^{\alpha-2}}{2(\alpha-2)}+\cdots\right], & 0<\alpha<2 \\
\frac{L^{3}}{4 G_{5}} \int d^{2} x \sqrt{1+\left(\partial_{c} w_{0}\right)^{2}}\left[-h_{0} \ln \left(\frac{\epsilon}{\ell}\right)+\cdots\right], & \alpha=2 . \\
\frac{L^{3}}{4 G_{5}} \int d^{2} x \sqrt{1+\left(\partial_{c} w_{0}\right)^{2}}(\text { finite terms }), & \alpha>2
\end{array}\right.
$$

The third counter term to cancel the remaining two divergences is composed of the scalar field $\left(\phi^{2}=-12 h_{0} z^{\alpha}+\cdots\right)$,

$$
S_{\mathrm{ct}}^{(3)}=\left\{\begin{array}{lr}
-\frac{\alpha L}{96(\alpha-2) G_{5}} \int d^{2} x \sqrt{\tilde{\gamma}} \phi^{2}, & 0<\alpha<2 \\
-\frac{L}{96 G_{5}} \ln \left(\frac{\epsilon}{\ell}\right) \int d^{2} x \sqrt{\tilde{\gamma}} \phi^{2}, & \alpha=2
\end{array} .\right.
$$

This counter term cancels all the divergences except for $\alpha<1$ where there are less divergent terms $\mathcal{O}\left(\epsilon^{2 \alpha-2}\right)$, which can be removed by counter terms containing higher powers of $\phi$ and $\tilde{K}$. Then from (2.42) and (2.23), one can define the renormalized HEEs under relevant perturbations for entangling regions in the asymptotically $\mathrm{AdS}_{5}$ geometry.

\subsection{Comments on the case of asymptotically $\mathrm{AdS}_{d+1>5}$ geometry}

As we increase the space-time dimensions, more diverging terms will enter the HEE formula. In order to exhaustively determine the forms of those divergences and fix the corresponding counter terms, one needs to solve the equation of motion of the embedding function $w\left(z, x^{a}\right)$ to greater order in $z$. In this subsection, to comment on the case of higher dimensions, we solve those equations up to quadratic order in $z$, which is enough to obtain the counter terms to cancel the three leading divergences in the renormalization of HEE in asymptotically $\operatorname{AdS}_{d+1}$ geometry, with $d>4$.

The asymptotically $\mathrm{AdS}_{d+1}$ metric in the Fefferman-Graham coordinates is given by

$$
d s^{2}=\frac{L^{2}}{z^{2}}\left(d z^{2}+(1+h(z)) \eta_{\mu \nu} d x^{\mu} d x^{\nu}\right) \quad \text { with } x^{\mu}=\left(t, x_{1}, x_{2}, \cdots, x_{d-2}, y\right) .
$$

The equations of motions for the metric fluctuation and the corresponding scalar field, which are derived from the action in (2.1), are solved by the following two independent solutions

$$
\begin{array}{lll}
\phi_{a}(z)=s_{0} z^{d-\Delta}+s_{1} z^{3(d-\Delta)}+\cdots & \Longrightarrow & h_{a}(z)=-\frac{s_{0}^{2}}{4(d-1)} z^{2(d-\Delta)}+h_{1} z^{4(d-\Delta)}+\cdots, \\
\phi_{b}(z)=v_{0} z^{\Delta}+v_{1} z^{3 \Delta}+\cdots & \Longrightarrow & h_{b}(z)=-\frac{v_{0}^{2}}{4(d-1)} z^{2 \Delta}+\tilde{h}_{1} z^{4 \Delta}+\cdots .
\end{array}
$$

As we did in the case of $d=3$ and $d=4$, we will use these solutions to determine the counter terms.

The RT hyper-surface $\Sigma_{A}$ is a $d$-1-dimensional manifold parametrized by the embedding

$$
x^{p}=\left(z, t=t_{0}, x_{1}, \cdots, x_{d-2}, y=w\left(z, x_{1}, \cdots, x_{d-2}\right)\right) .
$$


The induce metric on $\Sigma_{A}$ is given by

$$
\begin{aligned}
& \gamma_{z z}=\frac{L^{2}}{z^{2}}\left(1+w^{\prime 2}(1+h)\right), \quad \gamma_{z a}=\frac{L^{2}}{z^{2}} \partial_{a} w w^{\prime}(1+h), \\
& \gamma_{a b}=\frac{L^{2}}{z^{2}}(1+h)\left(\delta_{a b}+\partial_{a} w \partial_{b} w\right),
\end{aligned}
$$

where $\partial_{a}=\left(\partial_{x_{1}}, \cdots, \partial_{x_{d-2}}\right), w^{\prime}=\partial_{z} w$. Then the HEE is determined by the minimum value of the area of this hyper-surface given by

$$
\mathcal{A}_{\Sigma_{A}}=L^{d-1} \int d^{2} x \int d z \frac{(1+h)^{\frac{d-2}{2}}}{z^{d-1}} \sqrt{1+\left(\partial_{a} w\right)^{2}+(1+h) w^{\prime 2}} .
$$

The minimum area equation derived from this action is

$$
\begin{aligned}
& z(1+h) w^{\prime \prime}+z \partial_{a}^{2} w-(d-1)(1+h) w^{\prime}+\frac{d}{2} z h^{\prime} w^{\prime} \\
& -\frac{z}{1+\partial_{c} w^{2}+(1+h) w^{\prime 2}}\left[\partial_{a} w \partial_{b} w \partial_{a} \partial_{b} w+(1+h)^{2}\left(w^{\prime 2} w^{\prime \prime}+\frac{\frac{1}{2} h^{\prime} w^{\prime 3}+2 w^{\prime} \partial_{a} w \partial_{a} w^{\prime}}{1+h}\right)\right]=0
\end{aligned}
$$

We introduce the asymptotic expansion of $w\left(z, x^{a}\right)$ and solve this equation order by order in $z$ and we read the asymptotic expansion of $h(z)$ from (2.45),

$$
\begin{aligned}
h(z) & =h_{0} z^{\alpha}+\cdots, \\
w\left(z, x^{a}\right) & =w_{0}\left(x^{a}\right)+w_{2}\left(x^{a}\right) z^{2}+\cdots,
\end{aligned}
$$

where $\alpha$ is the smaller of $2(d-\Delta)$ and $2 \Delta$. The $w_{2}$ in (2.50) is determined in terms of $w_{0}$ by solving the minimal area equation $(2.49)$ and is given by

$$
w_{2}=\frac{1}{2(d-2)}\left(\partial_{a}^{2} w_{0}-\frac{\partial_{a} w_{0} \partial_{b} w_{0} \partial_{a} \partial_{b} w_{0}}{1+\left(\partial_{c} w_{0}\right)^{2}}\right)
$$

Plugging the expansion (2.50) into (2.48) and introducing the cut-off surface $z=\epsilon$, the HEE is given by

$$
S_{A}^{\mathrm{reg}}=\frac{L^{d-1}}{4 G_{d+1}} \int d^{d-2} x \int_{\epsilon}^{z_{m}} d z \frac{\sqrt{1+\left(\partial_{c} w_{0}\right)^{2}}}{z^{d-1}}\left[1+\frac{2 w_{2}^{2}+\partial_{a} w_{0} \partial_{a} w_{2}}{1+\left(\partial_{c} w_{0}\right)^{2}} z^{2}+\frac{d-2}{2} h_{0} z^{\alpha}+\cdots\right]
$$

Integrating by parts the second term in the square bracket in (2.52) and then using the result in (2.51), we obtain

$$
S_{A}^{\mathrm{reg}}=\frac{L^{d-1}}{4 G_{d+1}} \int d^{d-2} x \int_{\epsilon}^{z_{m}} d z \sqrt{1+\left(\partial_{c} w_{0}\right)^{2}}\left[\frac{1}{z^{d-1}}-\frac{2(d-3) w_{2}^{2}}{\left(1+\left(\partial_{c} w_{0}\right)^{2}\right) z^{d-3}}+\frac{(d-2) h_{0}}{2 z^{d-1-\alpha}}+\cdots\right] .
$$

Then we can carry out the $z$ integration to obtain

$$
S_{A}^{\mathrm{reg}}=\frac{L^{d-1}}{4 G_{d+1}} \int d^{d-2} x \frac{\sqrt{1+\left(\partial_{c} w_{0}\right)^{2}}}{\epsilon^{d-2}}\left[\frac{1}{(d-2)}-\frac{2(d-3) w_{2}^{2} \epsilon^{2}}{(d-4)\left(1+\left(\partial_{c} w_{0}\right)^{2}\right)}+\frac{(d-2) h_{0} \epsilon^{\alpha}}{2(d-2-\alpha)}+\cdots\right] .
$$


Next we fix the counter terms which are composed of the invariant quantities of the scalar field $\phi$, the induced metric, and extrinsic curvature on the boundary space $\partial \Sigma_{A}^{\epsilon}$. In this case the boundary space is a $(d-2)$-dimensional hypersurface embedded in the cut-off $z=\epsilon$ as $x^{i}=\left(x^{a}, y=w\left(\epsilon, x^{a}\right)\right)$. Using this embedding we can calculate the induced metric and the extrinsic curvature and they are given by (2.37). Then using the asymptotic expansion in (2.50), the trace of the extrinsic curvature is given by

$$
\tilde{K}=\frac{\epsilon(1+h)^{-1 / 2}}{L \sqrt{1+\left(\partial_{c} w_{0}\right)^{2}}}\left(\partial_{a}^{2} w_{0}-\frac{\partial_{a} w_{0} \partial_{b} w_{0} \partial_{a} \partial_{b} w_{0}}{1+\left(\partial_{c} w_{0}\right)^{2}}\right)+\cdots=\frac{2(d-2) \epsilon(1+h)^{-1 / 2}}{L \sqrt{1+\left(\partial_{c} w_{0}\right)^{2}}} w_{2}+\cdots,
$$

where in the last step we have used the solution of $w_{2}$ in (2.51).

The first counter term that cancels the leading order divergence in (2.54) is

$$
\begin{aligned}
S_{\mathrm{ct}}^{(1)} & =-\frac{L}{4(d-2) G_{d+1}} \int d^{d-2} x \sqrt{\tilde{\gamma}} \\
& =-\frac{L^{d-1}}{4 G_{d+1}} \int d^{d-2} x \frac{\sqrt{1+\left(\partial_{c} w_{0}\right)^{2}}}{\epsilon^{d-2}}\left[\frac{1}{(d-2)}-\frac{2 w_{2}^{2} \epsilon^{2}}{\left(1+\left(\partial_{c} w_{0}\right)^{2}\right)}+\frac{h_{0} \epsilon^{\alpha}}{2}+\cdots\right] .
\end{aligned}
$$

Adding the counter term (2.56) to (2.54), we obtain

$$
S_{A}^{\mathrm{reg}}+S_{\mathrm{ct}}^{(1)}=\frac{L^{d-1}}{4 G_{d+1}} \int d^{d-2} x \frac{\sqrt{1+\left(\partial_{c} w_{0}\right)^{2}}}{\epsilon^{d-2}}\left[-\frac{2 w_{2}^{2} \epsilon^{2}}{(d-4)\left(1+\left(\partial_{c} w_{0}\right)^{2}\right)}+\frac{\alpha h_{0} \epsilon^{\alpha}}{2(d-2-\alpha)}+\cdots\right] .
$$

The next required counter term is

$$
\begin{aligned}
S_{\mathrm{ct}}^{(2)} & =\frac{L^{3}}{8(d-2)^{2}(d-4) G_{d+1}} \int d^{d-2} x \sqrt{\tilde{\gamma}} \tilde{K}^{2} \\
& =\frac{L^{d-1}}{4 G_{d+1}} \int d^{d-2} x \frac{\sqrt{1+\left(\partial_{c} w_{0}\right)^{2}}}{\epsilon^{d-2}}\left[\frac{2 w_{2}^{2} \epsilon^{2}}{(d-4)\left(1+\left(\partial_{c} w_{0}\right)^{2}\right)}+\cdots\right] .
\end{aligned}
$$

Here we would like to note that there are more of such matter field independent divergences for $d>4$. For even $d$, the least divergent of those is the universal logarithmic divergence [40], whereas for odd $d$, it is the linear divergence. These are always cancelled by the counter terms built from the curvature invariants on $\partial \Sigma_{A}^{\epsilon}$ [35]. Now, adding the counter term (2.58) to (2.57), we obtain

$$
S_{A}^{\mathrm{reg}}+S_{\mathrm{ct}}^{(1)}+S_{\mathrm{ct}}^{(2)}=\frac{L^{d-1}}{4 G_{d+1}} \int d^{d-2} x \frac{\sqrt{1+\left(\partial_{c} w_{0}\right)^{2}}}{\epsilon^{d-2}}\left[\frac{\alpha h_{0} \epsilon^{\alpha}}{2(d-2-\alpha)}+\cdots\right] .
$$

The third counter term to cancel the next order divergences is composed of the scalar field $\left(\phi^{2}=-4(d-1) h_{0} z^{\alpha}+\cdots\right)$,

$$
\begin{aligned}
S_{\mathrm{ct}}^{(3)} & =\frac{\alpha L}{32(d-1)(d-2-\alpha) G_{d+1}} \int d^{d-2} x \sqrt{\tilde{\gamma}} \phi^{2} \\
& =-\frac{L^{d-1}}{4 G_{d+1}} \int d^{d-2} x \frac{\sqrt{1+\left(\partial_{c} w_{0}\right)^{2}}}{\epsilon^{d-2}}\left[\frac{\alpha h_{0} \epsilon^{\alpha}}{2(d-2-\alpha)}+\cdots\right] .
\end{aligned}
$$


This demonstrates, though there are numerous divergences in the case of higher dimensions, one can still construct the necessary counter terms from invariant quantities built from the scalar field $\phi$ and curvature invariants on the $(d-2)$-dimensional boundary of the regularized RT minimal area hypersurface. See appendix A for the completed renormalization of HEE in the simple case of pure $\mathrm{AdS}_{d+1}$ geometry with the disc entangling region.

\section{Renormalized HSC under relevant perturbations}

The CV conjecture for the subregion complexity [10] states that the HSC is equal to the volume of the codimension-one hypersurface $\mathcal{B}_{A}$ enclosed by the entangling subregion $A$ and the corresponding RT surface $\Sigma_{A}[4,5]$, i.e.,

$$
\mathcal{C}_{A}=\frac{V\left(\mathcal{B}_{A}\right)}{8 \pi L G_{d+1}}
$$

where $L$ is the radius of the $\mathrm{AdS}_{d+1}$ geometry. The CA conjecture for the subregion complexity was also proposed in [12]. In this section, we construct the renormalized HSC with entangling regions denoted by (2.7) for the asymptotically $\mathrm{AdS}_{4,5}$ geometries under relevant perturbations. To do that, one has to consider divergent terms generated from the boundary of the entangling region $\partial A$, which is different from the renormalization of the holographic complexity for the whole space [38].

\subsection{Renormalized HSC in asymptotically $\mathrm{AdS}_{4}$ geometry}

According to proposal of the HSC [10], we use the RT surface $\Sigma_{A}$ for entangling regions denoted by (2.7), which were obtained in the previous section. To regularize divergences of the HSC $\mathcal{C}_{A}$ in (3.1), we also introduce the $z=\epsilon$ cut-off, and then the regularized HSC is written as

$$
\mathcal{C}_{A}^{\mathrm{reg}}=\frac{1}{8 \pi L G_{4}} \int d x \int_{\epsilon}^{z_{m}} d z \int_{0}^{w(z, x)} d y \frac{L^{3}}{z^{3}}(1+h)=\frac{L^{2}}{8 \pi G_{4}} \int d x \int_{\epsilon}^{z_{m}} d z \frac{w(z, x)}{z^{3}}(1+h),
$$

where we used the asymptotically $\mathrm{AdS}_{4}$ geometry in $(2.2)$ and $w(z, x)$ is defined in the embedding (2.8). Using the asymptotic expansion (2.12), we obtain

$$
\mathcal{C}_{A}^{\mathrm{reg}}=\frac{L^{2}}{8 \pi G_{4}} \int d x \int_{\epsilon}^{z_{m}} d z\left(w_{0} z^{-3}+w_{2} z^{-1}+h_{0} w_{0} z^{\alpha-3}+\mathcal{O}\left(\epsilon^{2 \alpha-3}\right)+\cdots+\mathcal{O}\left(z^{\alpha-1}\right)\right) .
$$

Evaluating the $z$ integral, we single out the divergent terms as follows:

$$
\mathcal{C}_{A}^{\mathrm{reg}}=\left\{\begin{array}{lr}
\frac{L^{2}}{8 \pi G_{4}} \int d x\left(\frac{w_{0}}{2 \epsilon^{2}}-w_{2} \ln \left(\frac{\epsilon}{\ell}\right)+\frac{h_{0} w_{0} \epsilon^{\alpha-2}}{(2-\alpha)}+\frac{h_{1} w_{0} \epsilon^{2 \alpha-2}}{2(1-\alpha)}+\cdots\right), 0<\alpha<2 \\
\frac{L^{2}}{8 \pi G_{4}} \int d x\left(\frac{w_{0}}{2 \epsilon^{2}}-\left(w_{2}+h_{0} w_{0}\right) \ln \left(\frac{\epsilon}{\ell}\right)+\cdots\right), & \alpha=2, \\
\frac{L^{2}}{8 \pi G_{4}} \int d x\left(\frac{w_{0}}{2 \epsilon^{2}}-w_{2} \ln \left(\frac{\epsilon}{\ell}\right)+\cdots\right), & \alpha>2
\end{array}\right.
$$


where the relations between $\alpha$ and the conformal dimension $\Delta$ were given in (2.17). For $\alpha=1$ the last divergence in the first line will be logarithmic. We note that in the case of $\alpha=2$, there is only one divergence that depends on the scalar deformation whereas those divergences are absent when $\alpha>2$.

For a $(d+1)$-dimensional bulk space-time, the counter terms are given by

$$
\mathcal{C}_{\mathrm{ct}}=\int_{\partial \mathcal{M}_{\epsilon}} d^{d-1} x \sqrt{\operatorname{det} h_{i j}} \sum_{n} \mathcal{C}_{n}\left(g_{\mu \nu}, R_{\mu \nu}, h_{i j}, K_{i j}, \phi\right),
$$

where $\partial \mathcal{M}_{\epsilon}$ is a codimension-two static hyper-surface at the cut-off boundary $(z=\epsilon), h_{i j}$ is the induced metric on $\partial \mathcal{M}_{\epsilon}$, and $K_{i j}$ is the extrinsic curvature of the $\partial \mathcal{M}_{\epsilon}$ embedded in the bulk constant time slice $\mathcal{M}_{\epsilon}$. In addition, $g_{\mu \nu}$ is the induced metric on the cut-off $z=\epsilon$ boundary, $R_{\mu \nu}$ is the Ricci tensor derived from $g_{\mu \nu}$, and $\mathcal{C}_{n}$ are invariants built from $R_{\mu \nu}, g_{\mu \nu}, h_{i j}$, and $K_{i j}$ with appropriate mass dimensions. In the case we are considering, both $g_{\mu \nu}$ and $h_{i j}$ at $z=\epsilon$ are flat, i.e.,

$$
g_{\mu \nu}=\frac{L^{2}}{\epsilon^{2}}[1+h(\epsilon)] \eta_{\mu \nu}, \quad h_{i j}=\frac{L^{2}}{\epsilon^{2}}[1+h(\epsilon)] \delta_{i j},
$$

where $\mu, \nu=(t, x, y)$ and $i, j=(x, y)$. Therefore, the Ricci tensor $R_{\mu \nu}$ is vanishing and the extrinsic curvature is given by

$$
K_{i j}=L\left(\frac{h^{\prime}(\epsilon)}{2 \epsilon}-\frac{1+h(\epsilon)}{\epsilon^{2}}\right) \delta_{i j}, \quad K_{i z}=0, \quad K_{z z}=0 .
$$

According to the general formula in (3.5), the counter term which cancels the leading order divergence in (3.4) is given by

$$
\mathcal{C}_{\mathrm{ct}}^{(1)}=-\frac{1}{16 \pi G_{4}} \int d x \int_{0}^{w(\epsilon, x)} d y \sqrt{\operatorname{det} h_{i j}}=-\frac{L^{2}}{16 \pi G_{4}} \int d x\left(\frac{w_{0}}{\epsilon^{2}}+\frac{h_{0} w_{0}}{\epsilon^{2-\alpha}}+\frac{h_{1} w_{0}}{\epsilon^{2-2 \alpha}}+\cdots\right) .
$$

Since the leading order divergence in (3.4) is independent of the matter deformation, there is no contribution from the scalar field to the counter term at this order. Adding this leading counter term to the HSC in (3.4), we obtain

$$
\mathcal{C}_{A}^{\mathrm{reg}}+\mathcal{C}_{\mathrm{ct}}^{(1)}=\left\{\begin{array}{lr}
\frac{L^{2}}{8 \pi G_{4}} \int d x\left(-w_{2} \ln \left(\frac{\epsilon}{\ell}\right)+\frac{\alpha h_{0} w_{0} \epsilon^{\alpha-2}}{2(2-\alpha)}+\frac{\alpha h_{1} w_{0} \epsilon^{2 \alpha-2}}{2(1-\alpha)}+\cdots\right), & 0<\alpha<2 \\
\frac{L^{2}}{8 \pi G_{4}} \int d x\left(-w_{2} \ln \left(\frac{\epsilon}{\ell}\right)-h_{0} w_{0} \ln \left(\frac{\epsilon}{\ell}\right)+\cdots\right), & \alpha=2 . \\
\frac{L^{2}}{8 \pi G_{4}} \int d x\left(-w_{2} \ln \left(\frac{\epsilon}{\ell}\right)+\cdots\right), & \alpha>2
\end{array}\right.
$$

The $w_{2} \ln (\epsilon / \ell)$ divergence is also independent of the perturbations by the scalar field and it is present in the case of pure $\mathrm{AdS}_{4}$ background as well. We will come back to renormalization of this term later. First, let us discuss the other divergences. Since the Ricci tensor $R_{\mu \nu}$ is vanishing, the counter terms to cancel these other divergences are built from the invariants of $K_{i j}$ and $h_{i j}$, as well as the scalar field. We notice that, the contributions from 
the counter terms built from the curvature invariants on $\partial \mathcal{M}_{\epsilon}$ can be expressed in terms of the scalar field by using the solutions to the Einstein equation and the equation of motion of the scalar field obtained in (2.4). Therefore, we can express those counter terms purely in terms of the scalar field. Then, the counter term that has the right structure to cancel the second terms in the first and second lines of (3.9) is

$$
\mathcal{C}_{\mathrm{ct}}^{(2)}=\left\{\begin{array}{l}
-\frac{\alpha}{128 \pi G_{4}(\alpha-2)} \int d x \int_{0}^{w(\epsilon, x)} d y \sqrt{\operatorname{det} h_{i j}} \phi^{2}, \quad 0<\alpha<2 \\
-\frac{1}{64 \pi G_{4}} \ln \left(\frac{\epsilon}{\ell}\right) \int d x \int_{0}^{w(\epsilon, x)} d y \sqrt{\operatorname{det} h_{i j}} \phi^{2}, \quad \alpha=2
\end{array} .\right.
$$

Then we obtain

$$
\begin{aligned}
& \mathcal{C}_{A}^{\mathrm{reg}}+\mathcal{C}_{\mathrm{ct}}^{(1)}+\mathcal{C}_{\mathrm{ct}}^{(2)} \\
& =\left\{\begin{array}{lr}
\frac{L^{2}}{8 \pi G_{4}} \int d x\left(-w_{2} \ln \left(\frac{\epsilon}{\ell}\right)-\left(\frac{h_{1}}{2(\alpha-1)}-\frac{\left.4 h_{0}^{2}-\phi_{0} \phi_{1}\right)}{8(\alpha-2)}\right) \alpha w_{0} \epsilon^{2 \alpha-2}+\cdots\right), & 0<\alpha<2 \\
\frac{L^{2}}{8 \pi G_{4}} \int d x\left(-w_{2} \ln \left(\frac{\epsilon}{\ell}\right)+\cdots\right), & \alpha=2, \\
\frac{L^{2}}{8 \pi G_{4}} \int d x\left(-w_{2} \ln \left(\frac{\epsilon}{\ell}\right)+\cdots\right), & \alpha>2
\end{array}\right.
\end{aligned}
$$

where $\phi_{0}$ and $\phi_{1}$ are respectively, the coefficients of $z^{\alpha / 2}$ and $z^{3 \alpha / 2}$ in the scalar field solution in (2.4). From (3.11), we see that there is a divergence which is of order $\epsilon^{2 \alpha-2}$ for $\alpha<1$. In order to cancel this divergence we need to add counter terms that contain $K^{2}$ and $\phi^{4}$. Note that $K^{i j} K_{i j}$ also has the right order of divergence, however, since in our case $K^{i j} K_{i j}=\frac{1}{2} K^{2}$, it is not an independent contribution. Therefore, again replacing the $K^{2}$ contributions with the scalar field, the required counter term at this order is

$$
\mathcal{C}_{\mathrm{ct}}^{(3)}=\frac{C_{3}}{8 \pi G_{4}} \int d x \int_{0}^{w(\epsilon, x)} d y \sqrt{\operatorname{det} h_{i j}} \phi^{4}, \quad 0<\alpha<1,
$$

where $C_{3}=\frac{\alpha}{2^{7} h_{0}^{2}}\left(\frac{h_{1}}{\alpha-1}-\frac{\left.4 h_{0}^{2}-\phi_{0} \phi_{1}\right)}{4(\alpha-2)}\right)$. Adding the counter term (3.12) to (3.11), we obtain

$$
\mathcal{C}_{A}^{\mathrm{reg}}+\mathcal{C}_{\mathrm{ct}}^{(1)}+\mathcal{C}_{\mathrm{ct}}^{(2)}+\mathcal{C}_{\mathrm{ct}}^{(3)}=\left\{\begin{array}{lr}
\frac{L^{2}}{8 \pi G_{4}} \int d x\left(-w_{2} \ln \left(\frac{\epsilon}{\ell}\right)+\mathcal{O}\left(\epsilon^{3 \alpha-2}\right)+\cdots\right), & 0<\alpha<2 \\
\frac{L^{2}}{8 \pi G_{4}} \int d x\left(-w_{2} \ln \left(\frac{\epsilon}{\ell}\right)+\cdots\right), & \alpha=2 . \\
\frac{L^{2}}{8 \pi G_{4}} \int d x\left(-w_{2} \ln \left(\frac{\epsilon}{\ell}\right)+\cdots\right), & \alpha>2
\end{array}\right.
$$

Similarly, less divergent terms which are $\mathcal{O}\left(\epsilon^{n \alpha-2}\right)$ are removed by adding counter terms that are higher order in $K$ and $\phi$. However, the $w_{2} \ln (\epsilon / \ell)$ term, which is independent of the scalar deformation and it exists for all the three ranges of $\alpha$, can not be cancelled by any counter terms built from the invariants of $R_{\mu \nu}, g_{\mu \nu}, h_{i j} K_{i j}$, and $\phi$. Therefore, the general form of the counter terms (3.5) proposed in the literature does not account for this particular divergence. It turns out, these divergences are cancelled by counter terms which are built from the invariants of the induced metric $\tilde{\gamma}_{a b}$ and the extrinsic curvature 
$\tilde{K}_{a b}$ on the boundary $\partial \Sigma_{A}^{\epsilon}$ of the regularized minimal surface $\Sigma_{A}^{\epsilon}$ [12]. In our case these induced metric and the extrinsic curvature are as in (2.18). The required counter term which cancels the logarithmic divergence in (3.13) is

$$
\tilde{\mathcal{C}}_{\mathrm{ct}}=\frac{L^{2}}{16 \pi G_{4}} \ln \left(\frac{\epsilon}{\ell}\right) \int d x \sqrt{\operatorname{det} \tilde{\gamma}_{x x}} \tilde{K}=\frac{L^{2}}{8 \pi G_{4}} \ln \left(\frac{\epsilon}{\ell}\right) \int d x\left(w_{2}+\mathcal{O}\left(\epsilon^{2}\right)\right) .
$$

Adding this counter term to (3.13), we finally obtain a finite result for $\alpha \geq \frac{2}{3}$ whereas for $\alpha<\frac{2}{3}$, as it was stated above, we need more counter terms containing higher powers of $K$ and $\phi$. However, we note that the number of the necessary counter terms are finite once one fixes the value of $\alpha$, therefore, in the case of asymptotically $\mathrm{AdS}_{4}$ geometry, HSC is renormalizable for any value of $\alpha$.

\subsection{Renormalized $\mathrm{HSC}$ in asymptotically $\mathrm{AdS}_{5}$ geometry}

The renormalization of HSC in asymptotically $\mathrm{AdS}_{5}$ geometry follows the same steps as the $d=3$ case, however, in the current case there is an extra $\mathcal{O}\left(\epsilon^{\alpha-1}\right)$ divergence which can not be cancelled by any of the counter terms listed in the previous subsection. The regularized HSC is given by

$$
\mathcal{C}_{A}^{\mathrm{reg}}=\frac{L^{3}}{8 \pi G_{5}} \int d^{2} x \int_{\epsilon}^{z_{m}} d z(1+h)^{\frac{3}{2}} \frac{w\left(z, x_{1}, x_{2}\right)}{z^{4}} .
$$

Evaluating the $z$ integration in (3.15), we obtain

$$
\mathcal{C}_{A}^{\mathrm{reg}}=\left\{\begin{array}{lr}
\frac{L^{3}}{8 \pi G_{5}} \int d^{2} x\left(\frac{w_{0}}{3 \epsilon^{3}}+\frac{w_{2}}{\epsilon}+\frac{3}{2(3-\alpha)} \frac{w_{0} h_{0}}{\epsilon^{3-\alpha}}+\frac{3 h_{0} w_{2}+2 w_{\alpha}+2}{2(1-\alpha) \epsilon^{1-\alpha}}+\mathcal{O}\left(\epsilon^{2 \alpha-3}\right)+\cdots\right), & 0<\alpha<2 \\
\frac{L^{3}}{8 \pi G_{5}} \int d^{2} x\left(\frac{w_{0}}{3 \epsilon^{3}}+\frac{w_{2}}{\epsilon}+\frac{3}{2(3-\alpha)} \frac{w_{0} h_{0}}{\epsilon^{3-\alpha}}+\cdots\right), & \alpha \geq 2
\end{array},\right.
$$

where the relations between $\alpha$ and the conformal dimension $\Delta$ were given in (2.32). Note that for $\alpha=1$ and $\alpha=3$ cases, we get logarithmic divergences and those cases should be treated separately as in the previous subsection.

As in the previous subsection, the Ricci tensor derived from the flat boundary metric $g_{\mu \nu}=\frac{L^{2}}{\epsilon^{2}}[1+h(\epsilon)] \eta_{\mu \nu}$ is vanishing. Therefore, the counter terms are obtained from the induced metric $h_{i j}$, the extrinsic curvature $K_{i j}$ on the cut-off surface $z=\epsilon$, and the scalar field. The induced metric and the extrinsic curvature are

$$
h_{i j}=\frac{L^{2}}{\epsilon^{2}}[1+h(\epsilon)] \delta_{i j}, \quad K_{i j}=\frac{L}{2 \epsilon^{2}}\left[\epsilon h^{\prime}(\epsilon)-2 h(\epsilon)-2\right] \delta_{i j},
$$

where $i, j=\left(x_{1}, x_{2}, y\right)$ are the coordinates on the cut-off surface at fixed time $t=t_{0}$. The appropriate counter term which removes the leading divergence in (3.16) is

$$
\mathcal{C}_{\mathrm{ct}}^{(1)}=-\frac{1}{24 \pi G_{5}} \int d^{2} x \sqrt{\operatorname{det} h_{i j}} w\left(\epsilon, x_{1}, x_{2}\right) .
$$


Adding this counter term to (3.16), we obtain

$$
\mathcal{C}_{A}^{\mathrm{reg}}+\mathcal{C}_{\mathrm{ct}}^{(1)}=\left\{\begin{array}{lr}
\frac{L^{3}}{8 \pi G_{5}} \int d^{2} x\left(\frac{2 w_{2}}{3 \epsilon}+\frac{\alpha}{2(3-\alpha)} \frac{w_{0} h_{0}}{\epsilon^{3-\alpha}}+\frac{(\alpha+2)\left(3 h_{0} w_{2}+2 w_{\alpha+2}\right)}{6(1-\alpha) \epsilon^{1-\alpha}}+\cdots\right), & 0<\alpha<2 \\
\frac{L^{3}}{8 \pi G_{5}} \int d^{2} x\left(\frac{2 w_{2}}{3 \epsilon}+\frac{\alpha}{2(3-\alpha)} \frac{w_{0} h_{0}}{\epsilon^{3-\alpha}}+\cdots\right), & \alpha \geq 2
\end{array}\right.
$$

The counter term that cancels $\mathcal{O}\left(\epsilon^{\alpha-3}\right)$ divergence is linear in $K$ and quadratic in $\phi$. Replacing the contribution from the extrinsic curvature with the scalar field by using the gravity equations of motion, we can write

$$
\mathcal{C}_{\mathrm{ct}}^{(2)}=-\frac{\alpha}{192 \pi G_{5}(\alpha-3)} \int d^{2} x \int_{0}^{w(\epsilon, x)} d y \sqrt{\operatorname{det} h_{i j}} \phi^{2} .
$$

Adding this counter term to (3.19), we obtain

$$
\mathcal{C}_{A}^{\mathrm{reg}}+\mathcal{C}_{\mathrm{ct}}^{(1)}+\mathcal{C}_{\mathrm{ct}}^{(2)}=\left\{\begin{array}{lr}
\frac{L^{3}}{8 \pi G_{5}} \int d^{2} x\left(\frac{2 w_{2}}{3 \epsilon}+\frac{9 h_{0} w_{2}+\left(6+\alpha-\alpha^{2}\right) w_{\alpha+2}}{3(\alpha-3)(\alpha-1) \epsilon^{1-\alpha}}+\cdots\right), & 0<\alpha<1, \\
\frac{L^{3}}{8 \pi G_{5}} \int d^{2} x\left(\frac{2 w_{2}}{3 \epsilon}+\cdots\right), & \alpha>1
\end{array}\right.
$$

where we notice that the ranges of $\alpha$ are changed from those of (3.19) and the corresponding ranges of the conformal dimension $\Delta$ are

$$
\begin{aligned}
0<\alpha<1 & \Longleftrightarrow 0<\Delta<\frac{1}{2} \text { and } \frac{7}{2}<\Delta<4, \\
\alpha>1 & \Longleftrightarrow \frac{1}{2}<\Delta<\frac{7}{2} .
\end{aligned}
$$

Like it was in the previous subsection, there is a divergence which is independent from the scalar field deformation and it is $\mathcal{O}\left(\epsilon^{-1}\right)$. In order to cancel this divergence we need to introduce the extrinsic curvature on the boundary $\partial \Sigma_{A}^{\epsilon}$ of the RT surface, as we did in the previous subsection. This corresponding extrinsic curvature was given in (2.38). Therefore, the counter term that cancels the $\mathcal{O}\left(\epsilon^{-1}\right)$ divergence in (3.21) is given by

$$
\tilde{\mathcal{C}}_{\mathrm{ct}}=-\frac{L^{2}}{48 \pi G_{5}} \int d^{2} x \sqrt{\operatorname{det} \tilde{\gamma}_{a b}} \tilde{K}=-\frac{L^{3}}{8 \pi G_{5}} \int d^{2} x\left(\frac{2 w_{2}}{3 \epsilon}+\frac{h_{0} w_{2}}{3 \epsilon^{1-\alpha}}+\mathcal{O}(\epsilon)\right) .
$$

Adding this counter term to (3.21), we finally obtain

$$
\mathcal{C}_{A}^{\mathrm{reg}}+\mathcal{C}_{\mathrm{ct}}^{(1,2)}+\tilde{\mathcal{C}}_{\mathrm{ct}}=\left\{\begin{array}{lr}
\frac{L^{3}}{8 \pi G_{5}} \int d^{2} x\left(\frac{\left(6+4 \alpha-\alpha^{2}\right) h_{0} w_{2}+\left(6+\alpha-\alpha^{2}\right) w_{\alpha+2}}{3(\alpha-3)(\alpha-1) \epsilon^{1-\alpha}}+\cdots\right), & 0<\alpha<1 \\
\frac{L^{3}}{8 \pi G_{5}} \int d^{2} x\left(\mathcal{O}\left(\epsilon^{2 \alpha-3}\right)+\cdots\right), & \alpha>1
\end{array} .\right.
$$

Next we need to remove the $\mathcal{O}\left(\epsilon^{\alpha-1}\right)$ divergence. The possible counter term is

$$
\mathcal{C}_{\mathrm{ct}}^{(3)}=\frac{B_{\alpha}}{8 \pi G_{5}} \int d^{2} x \int_{0}^{w(\epsilon, x)} d y \sqrt{\operatorname{det} h_{i j}}\left(\phi^{2}+C_{\alpha} \phi^{4}\right)
$$


This counter term produces unwanted divergent term, which is $\mathcal{O}\left(\epsilon^{\alpha-3}\right)$, in addition to the wanted $\mathcal{O}\left(\epsilon^{\alpha-1}\right)$ divergent term. Then, we could fix the values of the undetermined constants $B_{\alpha}$ and $C_{\alpha}$ by setting the coefficient of the unwanted divergence to zero. However, the coefficient of the $\mathcal{O}\left(\epsilon^{\alpha-3}\right)$ term is the same as that of $\mathcal{O}\left(\epsilon^{\alpha-1}\right)$ term, which means setting the former to zero does eliminate the later as well. In general, it is impossible to write a counter term for the $\mathcal{O}\left(\epsilon^{\alpha-1}\right)$ divergence without producing the $\mathcal{O}\left(\epsilon^{\alpha-3}\right)$ unwanted divergence. Therefore, after fixing the undetermined constants in (3.25), we can cancel the less divergent term $\mathcal{O}\left(\epsilon^{2 \alpha-3}\right)$ but can not cancel the $\mathcal{O}\left(\epsilon^{\alpha-1}\right)$ divergence. Hence, the $\mathcal{O}\left(\epsilon^{\alpha-1}\right)$ divergence can not be removed, which means there is no renormalized HSC in the range $0<\alpha<1$, i.e., $0<\Delta<\frac{1}{2}$ and $\frac{7}{2}<\Delta<4$. Here the latter case does not violate the unitary bound $(\Delta \geq 1)$ for primary operators in 4 -dimensional dual field theory. It will be interesting if one figures out the physical reason of this phenomenon by investigating other HSC conjectures or spacetime dimensions.

\subsection{Comments on the case of asymptotically $\operatorname{AdS}_{d+1>5}$ geometry}

In order to comment on the deliberation of the renoramalization of HSC in general spacetime dimensions, in this section, we show the cancellation of the leading three divergences for any $d>4$. Using the general definition in (3.1), the regularized HSC in asymptotically $\mathrm{AdS}_{d+1}$ geometry is given by

$$
\mathcal{C}_{A}^{\mathrm{reg}}=\frac{L^{d-1}}{8 \pi G_{d+1}} \int d^{d-2} x \int_{\epsilon}^{z_{m}} d z(1+h)^{\frac{d-1}{2}} \frac{w\left(z, x^{a}\right)}{z^{d}} .
$$

Evaluating the $z$ integration in (3.26), we obtain

$$
\mathcal{C}_{A}^{\mathrm{reg}}=\frac{L^{d-1}}{8 \pi G_{d+1}} \int \frac{d^{d-2} x}{\epsilon^{d-1}}\left(\frac{w_{0}}{d-1}+\frac{w_{2} \epsilon^{2}}{d-3}+\frac{(d-1) w_{0} h_{0} \epsilon^{\alpha}}{2(d-1-\alpha)}+\cdots\right)
$$

The counter terms that cancels the first and the third divergences in (3.27) are obtained from the induced metric $h_{i j}$ and the extrinsic curvature $K_{i j}$ on the cut-off surface $z=\epsilon$, which are given by

$$
h_{i j}=\frac{L^{2}}{\epsilon^{2}}[1+h(\epsilon)] \delta_{i j}, \quad K_{i j}=\frac{L}{2 \epsilon^{2}}\left[\epsilon h^{\prime}(\epsilon)-2 h(\epsilon)-2\right] \delta_{i j},
$$

where $i, j=\left(x^{a}, y\right)$ are the coordinates on the cut-off surface at fixed time $t=t_{0}$. The appropriate counter term which removes the leading divergence in (3.27) is

$$
\mathcal{C}_{\mathrm{ct}}^{(1)}=-\frac{1}{8(d-1) \pi G_{d+1}} \int d^{d-2} x \int_{0}^{w\left(\epsilon, x^{a}\right)} d y \sqrt{\operatorname{det} h_{i j}}
$$

Adding this counter term to (3.27), we obtain

$$
\mathcal{C}_{A}^{\mathrm{reg}}+\mathcal{C}_{\mathrm{ct}}^{(1)}=\frac{L^{d-1}}{8 \pi G_{d+1}} \int d^{d-2} x\left(\frac{2 w_{2}}{(d-1)(d-3) \epsilon^{d-3}}+\frac{\alpha}{2(d-1-\alpha)} \frac{w_{0} h_{0}}{\epsilon^{d-1-\alpha}}+\cdots\right)
$$


The counter term that cancels $\mathcal{O}\left(\epsilon^{\alpha+1-d}\right)$ divergence is linear in $K$ and quadratic in $\phi$ and is given by

$$
\mathcal{C}_{\mathrm{ct}}^{(2)}=\frac{\alpha}{64 \pi G_{d+1}(d-1)(d-1-\alpha)} \int d^{d-2} x \int_{0}^{w(\epsilon, x)} d y \sqrt{\operatorname{det} h_{i j}} \phi^{2},
$$

where we have replaced the $K$ term using the equations of motion as usual. Adding this counter term to (3.30), we obtain

$$
\mathcal{C}_{A}^{\mathrm{reg}}+\mathcal{C}_{\mathrm{ct}}^{(1)}+\mathcal{C}_{\mathrm{ct}}^{(2)}=\frac{L^{d-1}}{8 \pi G_{d+1}} \int d^{d-2} x\left(\frac{2 w_{2}}{(d-1)(d-3) \epsilon^{d-3}}+\cdots\right)
$$

The next order divergence is $\mathcal{O}\left(\epsilon^{3-d}\right)$, which is independent from the scalar field deformation. In order to cancel this divergence, we need to introduce the extrinsic curvature on the boundary $\partial \Sigma_{A}^{\epsilon}$ of the RT surface. The corresponding extrinsic curvature was given in (2.55). Therefore, the counter term that cancels the $\mathcal{O}\left(\epsilon^{d-3}\right)$ divergence in (3.32) is given by

$$
\begin{aligned}
\tilde{\mathcal{C}}_{\mathrm{ct}} & =-\frac{L^{2}}{8 \pi(d-1)(d-2)(d-3) G_{d+1}} \int d^{d-2} x \sqrt{\operatorname{det} \tilde{\gamma}_{a b}} \tilde{K} \\
& =-\frac{L^{d-1}}{8 \pi G_{d+1}} \int d^{d-2} x\left(\frac{2 w_{2}}{(d-1)(d-3) \epsilon^{d-3}}+\cdots\right) .
\end{aligned}
$$

Though what we have done here is not complete, it is enough to see that in any dimensions, the counter terms needed to renormalize the HSC are expressed as integrals of the curvature invariants on the $(d-1)$-dimensional cut-off boundary plus integrals of the curvature invariants on the $(d-2)$-dimensional boundary of the RT hyper-surface. See appendix B for the fully renormalized HSC in the case of $\mathrm{AdS}_{d+1}$ geometry and disc entangling space.

\section{An example: the LLM geometry}

In this section, we test the general procedure we discussed in the previous sections, by using an asymptotically $\mathrm{AdS}_{4}$ geometry, which is obtained from the KK reduction of the 11-dimensional LLM solutions [39]. For definiteness, we choose the boundary subspace $A$ to be a disc of radius $R$.

\subsection{The LLM geometry}

We have presented a detailed account of the LLM geometries with (or without) discrete torsion and applied the KK holography procedure [41, 42] to obtain the vacuum expectation values (vevs) of chiral primary operators (CPOs) with conformal dimensions $\Delta=1,2$ in the $\mathrm{U}_{k}(N) \times \mathrm{U}_{-k}(N)$ mass-deformed ABJM $(\mathrm{mABJM})$ theory $[43,44] .{ }^{3}$ Here the mABJM theory is obtained from the supersymmetry preserving mass deformation of the $\mathcal{N}=6$ ABJM theory [48]. In this subsection, we briefly review some necessary aspects of the LLM geometries and the KK reduction to asymptotically $\mathrm{AdS}_{4}$ geometry.

\footnotetext{
${ }^{3}$ The gauge/gravity duality between the $\mathcal{N}=6$ supersymmetry preserving mABJM theory and the LLM geometry was investigated in the large $N$ limit [45-47].
} 
The LLM geometries with $\mathrm{SO}(2,1) \times \mathrm{SO}(4) / \mathbb{Z}_{k} \times \mathrm{SO}(4) / \mathbb{Z}_{k}$ isometry are BPS solutions of the 11-dimensional supergravity [39,49]. The metric and the corresponding 4-form field strength are given by

$$
\begin{aligned}
d s^{2}= & -G_{t t}\left(-d t^{2}+d w_{1}^{2}+d w_{2}^{2}\right)+G_{x x}\left(d \tilde{x}^{2}+d \tilde{y}^{2}\right)+G_{\theta \theta} d s_{S^{3} / \mathbb{Z}_{k}}^{2} G_{\tilde{\theta} \tilde{\theta}} d s_{\tilde{S}^{3} / \mathbb{Z}_{k}}^{2} \\
F_{4}= & -d\left(e^{2 \Phi} h^{-2} V\right) \wedge d t \wedge d w_{1} \wedge d w_{2}+\mu_{0}^{-1}\left[V d\left(\tilde{y}^{2} e^{2 G}\right)+h^{2} e^{3 G} \star_{2} d\left(\tilde{y}^{2} e^{-2 G}\right)\right] \wedge d \Omega_{3} \\
& +\mu_{0}^{-1}\left[V d\left(\tilde{y}^{2} e^{-2 G}\right)-h^{2} e^{-3 G} \star_{2} d\left(\tilde{y}^{2} e^{2 G}\right)\right] \wedge d \tilde{\Omega}_{3},
\end{aligned}
$$

where $\mu_{0}$ is a mass parameter, $d s_{S^{3} / \mathbb{Z}_{k}}^{2}$ and $d s_{\tilde{S}^{3} / \mathbb{Z}_{k}}^{2}$ are metrics of two $S^{3}$ 's with $\mathbb{Z}_{k}$ orbifold, while $d \Omega_{3}$ and $d \tilde{\Omega}_{3}$ are the corresponding volume forms. The metric $G_{p q}$ and the 4 -form field strength $F_{p q r s}$ are completely determined by the two functions $Z(\tilde{x}, \tilde{y})$ and $V(\tilde{x}, \tilde{y})$,

$$
Z(\tilde{x}, \tilde{y})=\sum_{i=1}^{2 N_{b}+1} \frac{(-1)^{i+1}\left(\tilde{x}-\tilde{x}_{i}\right)}{2 \sqrt{\left(\tilde{x}-\tilde{x}_{i}\right)^{2}+\tilde{y}^{2}}}, \quad V(\tilde{x}, \tilde{y})=\sum_{i=1}^{2 N_{b}+1} \frac{(-1)^{i+1}}{2 \sqrt{\left(\tilde{x}-\tilde{x}_{i}\right)^{2}+\tilde{y}^{2}}}
$$

where $\tilde{x}_{i}$ are the location of the boundaries between the black/white regions in the droplet representations of the geometries and $N_{b}$ is the number of black or white regions with finite lengths. See [45-47] for functional forms of $G_{p q}$ and $F_{p q r s}$ and other detailed conventions. These functions are written in terms of the Legendre polynomials as follows [50],

$$
\begin{aligned}
& Z(r, \xi)=\frac{1}{2}\left[\xi+\sum_{n=1}^{\infty} \mathrm{C}_{n}\left[(n+1) P_{n+1}(\xi)-2 \xi n P_{n}(\xi)+(n-1) P_{n-1}(\xi)\right]\left(\frac{2 \pi \mu_{0} l_{\mathrm{P}}^{3}}{r}\right)^{n}\right] \\
& V(r, \xi)=\frac{1}{2 r}\left[1+\sum_{n=1}^{\infty} \mathrm{C}_{n} P_{n}(\xi)\left(\frac{2 \pi \mu_{0} l_{\mathrm{P}}^{3}}{r}\right)^{n}\right]
\end{aligned}
$$

where $\xi=\frac{\tilde{x}}{r}$ with $r=\sqrt{\tilde{x}^{2}+\tilde{y}^{2}}, P_{n}(\xi)$ are the Legendre polynomials, and we have introduced [47]

$$
\mathrm{C}_{n}=\sum_{i=1}^{2 N_{b}+1}(-1)^{i+1}\left(\frac{\tilde{x}_{i}}{2 \pi \mu_{0} l_{\mathrm{P}}^{3}}\right)^{n}
$$

with Planck length $l_{\mathrm{P}}$. In order to obtain the HEE for the mABJM theory in the small mass limit, we consider the asymptotic expansion of the LLM geometries up to quadratic order in $\mu_{0} \cdot{ }^{4}$ One can see in this small mass limit that physical quantities, such as vevs of CPOs with $\Delta=1,2$ and the HEE, are completely expressed by the following two quantities

$$
A_{2}=\frac{1}{2}\left(\mathrm{C}_{2}-\mathrm{C}_{1}^{2}\right), \quad A_{3}=\frac{1}{3}\left(\mathrm{C}_{3}-3 \mathrm{C}_{1} \mathrm{C}_{2}+2 \mathrm{C}_{1}^{3}\right) .
$$

Before we write the non-linear KK reduction of the 11-dimensional supergravity, we notice that the $\mathbb{Z}_{k}$-orbifold has no non-trivial role in the KK reduction. The reason is the following. The LLM geometry has $\mathrm{SO}(2,1) \times \mathrm{SO}(4) / \mathbb{Z}_{k} \times \mathrm{SO}(4) / \mathbb{Z}_{k}$ isometry and becomes

\footnotetext{
${ }^{4}$ The HEEs for the massive ABJM theory was investigated in various contexts [46, 50-53].
} 
asymptotically $\mathrm{AdS}_{4} \times S^{7} / \mathbb{Z}_{k}$. In order to reflect such symmetry of the LLM geometry, one needs to write the asymptotic metric $S^{7} / \mathbb{Z}_{k}$ as

$$
d s_{S^{7} / \mathbb{Z}_{k}}^{2}=d \tau^{2}+\frac{d \theta^{2}+\sin ^{2} \theta d \phi^{2}+(d \psi+\cos \theta d \phi)^{2}}{4}+\frac{d \tilde{\theta}^{2}+\sin ^{2} \tilde{\theta} d \tilde{\phi}^{2}+(d \tilde{\psi}+\cos \tilde{\theta} d \tilde{\phi})^{2}}{4},
$$

where $(\theta, \phi, \psi)$ and $(\tilde{\theta}, \tilde{\phi}, \tilde{\psi})$ are Euler angles with ranges, $0 \leq \theta, \tilde{\theta} \leq \pi, 0 \leq \phi, \tilde{\phi} \leq 2 \pi$, and $0 \leq \psi, \tilde{\psi} \leq \frac{4 \pi}{k}[49,54]$. We see that in (4.7), the two $S^{1}$ circles with angles $\psi$ and $\tilde{\psi}$ are orbifolded. However, in the asymptotic expansion of the LLM geometries, components of the metric and the 4-form field strength have no dependence of $\psi$ and $\tilde{\psi}$. For this reason, one can follow the method of non-linear KK reduction developed in [45-47], even for the cases of $k>1$, since the presence of discrete torsion [47] is originated from the $\mathbb{Z}_{k}$-orbifold and is only related to the coordinates $\psi$ and $\tilde{\psi}$.

Keeping in mind the comments in the previous paragraph, one can implement the non-linear KK reduction up to quadratic order in $\mu_{0}$ for the LLM geometry to obtain an asymptotically $\mathrm{AdS}_{4}$ geometry. Then we obtain

$$
d s^{2}=\frac{L^{2}}{z^{2}}\left[f(z) \eta_{i j} d x^{i} d x^{j}+g(z) d z^{2}\right],
$$

where $\eta_{i j}=\operatorname{diag}(-1,1,1)$ and

$$
\begin{aligned}
& f(z)=1-\frac{1}{45}\left(30+\beta_{3}^{2}\right)\left(\mu_{0} z\right)^{2}+\mathcal{O}\left(z^{4}\right), \\
& g(z)=1-\frac{1}{360}\left(960+29 \beta_{3}^{2}\right)\left(\mu_{0} z\right)^{2}+\mathcal{O}\left(z^{4}\right) .
\end{aligned}
$$

Here, the quantities $\beta_{3}$ and the radius of the $\mathrm{AdS}_{4} L$ are written in terms of $A_{2}$ and $A_{3}$ as

$$
\beta_{3}=\frac{3 A_{3}}{A_{2}^{3 / 2}}, \quad L=\frac{1}{2}\left(32 \pi^{2} A_{2}\right)^{1 / 6} l_{\mathrm{P}}
$$

In order to write the metric in the FG coordinate, we introduce the coordinate transformation $z \rightarrow z+\frac{\mu_{0}^{2}}{1440}\left(960+29 \beta_{3}^{2}\right) z^{3}$. The result is

$$
d s^{2}=\frac{L^{2}}{z^{2}}\left[d z^{2}+\left(1-\left(2+\frac{\beta_{3}^{2}}{16}\right)\left(\mu_{0} z\right)^{2}+\mathcal{O}\left(\left(\mu_{0} z\right)^{4}\right)\right) \eta_{i j} d x^{i} d x^{j}\right] .
$$

In the next subsection, we use the asymptotically $\mathrm{AdS}_{4}$ metric in (4.11) to construct the renormalized HEE and HSC following the methods developed in the previous sections.

\subsection{Renormalized HEE and HSC in the mABJM theory}

In [46], we have shown that the metric in (4.11) is a solution to equations of motion derived from the action of Einstein gravity with negative cosmological constant coupled to two scalar fields $T$ and $\Psi$. The action was obtained from the KK reduction of the 11-dimensional gravity on LLM background, and it is given by

$$
S=\frac{1}{16 \pi G_{4}} \int d^{4} x \sqrt{-g}(\mathcal{R}-2 \Lambda)+S_{m},
$$


where $\Lambda=-\frac{3}{L^{2}}$ is the negative cosmological constant and the matter action is given by

$$
S_{m}=-\frac{1}{32 \pi G_{4}} \int d^{4} x \sqrt{-g}\left(\nabla_{p} T \nabla^{p} T+M_{t}^{2} T^{2}+\nabla_{p} \Psi \nabla^{p} \Psi+M_{\psi}^{2} \Psi^{2}\right) .
$$

The field $\Psi$ is a genuine scalar, which is dual to the CPO of conformal dimension $\Delta=1$, with mass $M_{\psi}^{2}=\frac{\Delta(\Delta-3)}{L^{2}}=-\frac{2}{L^{2}}$, whereas $T$ is a pseudoscalar and it is dual to a gauge invariant operator of conformal dimension $\Delta=2$, hence has the same mass as that of $\Psi$.

The solutions to the equations of motion of those scalar fields as well were obtained from the KK reduction of the 11-dimensional LLM solutions:

$$
T(z)=4 \mu_{0} z+s_{1} z^{3}+\cdots, \quad \Psi(z)=-\frac{1}{\sqrt{2}} \beta_{3} \mu_{0} z+v_{1} z^{3}+\cdots .
$$

Using the conformal dimensions assignments of the previous paragraph and comparing this solution with the general solutions we wrote in section 2.1, we notice that for the field $T$ the solutions belong to the first type of solution in (2.4) with $s_{0}=4 \mu_{0}$, whereas for the field $\Psi$ they belong to the second type of solutions in (2.4) with $v_{0}=-\frac{1}{\sqrt{2}} \beta_{3} \mu_{0}$. One can also read the solution to the warp factor $h(z)$ by using (2.4) and (4.14). The answer is

$$
h(z)=-\frac{s_{0}^{2}}{8} z^{2}+\cdots-\frac{v_{0}^{2}}{8} z^{2}+\cdots=-\left(2+\frac{\beta_{3}^{2}}{16}\right) \mu_{0}^{2} z^{2}+\cdots .
$$

As expected, this is consistent with the value of the warp factor that can be read from (4.11).

The parametrization in (2.8), which describe the embedding of the minimal surface $\Sigma_{A}$ into the bulk space, is convenient to separate the divergent terms from the regular terms and then propose the appropriate counter terms to cancel those divergences. For the case at hand, where the subspace $A$ is a disc of radius $R$, however, to calculate the finite value of the regularized HEE and HSC, one needs to find the re-summation of the series in (2.12) at each order in the mass parameter $\mu_{0}$, which is very difficult. Therefore, we introduce an alternative parametrization for the embedding as

$$
x^{p}=\left(t=t_{0}, z, x=\rho(z) \cos \theta, y=\rho(z) \sin \theta\right) .
$$

Though we choose the mapping (4.16), which is different from that of (2.8), the resulting counter terms for the HEE and the HSC have the same forms since they are independent of coordinate choices.

Denoting the coordinates on the minimal surface as $\sigma^{\alpha}=(\theta, \rho)$, the induced metric $\gamma_{\alpha \beta}=\partial_{\alpha} x^{p} \partial_{\beta} x^{q} g_{p q}$ becomes

$$
\gamma_{\rho \rho}=\frac{L^{2}}{z^{2}}\left[\frac{1}{\rho^{\prime}(z)^{2}}+(1+h(z))\right], \quad \gamma_{\theta \theta}=\frac{L^{2}}{z^{2}}(1+h(z)) \rho(z)^{2},
$$

where we have used the bulk metric $g_{p q}$ in (4.11) and the warp factor is read from (4.15)

$$
1+h(z)=1+h_{0} z^{\alpha}+h_{1} z^{2 \alpha}+\cdots, \quad \text { with } \alpha=2, h_{0}=-\left(2+\frac{\beta_{3}^{2}}{16}\right) \mu_{0}^{2} .
$$


The area of the minimal surface is given by

$$
\mathcal{A}_{\Sigma_{A}}=\int d \rho \int d \theta \sqrt{\operatorname{det} \gamma_{\alpha \beta}}=2 \pi L^{2} \int_{0}^{z_{m}} d z \mathcal{L}_{A},
$$

where

$$
\mathcal{L}_{A}=\frac{\rho(z)}{z^{2}} \sqrt{1+h(z)+\left((1+h(z)) \rho^{\prime}(z)\right)^{2}} .
$$

In order to determine $\rho(z)$ which minimize the area $\mathcal{A}_{\Sigma}$, we solve the Euler-Lagrangian equation for $\mathcal{L}_{A}$, order by order in $\mu_{0}$ with the boundary condition $\rho(z=0)=R$. Up to quadratic order in $\mu_{0}$, the result is $[46,50,51]$

$$
\rho(z)=\rho_{0}(z)+\rho_{2}(z) \mu_{0}^{2}+\mathcal{O}\left(\mu_{0}^{4}\right)
$$

where

$$
\begin{aligned}
& \rho_{0}(z)=\sqrt{R^{2}-z^{2}}, \\
& \rho_{2}(z)=\frac{\mu_{0}^{2}}{6 \sqrt{R^{2}-z^{2}}}\left(4+\frac{\beta_{3}^{2}}{8}\right)\left[-\frac{z^{4}}{2}+2 R^{2} z^{2}-4 R^{3} z+4 R^{4} \log \left(\frac{R+z}{R}\right)\right] .
\end{aligned}
$$

The value of the turning point $z_{m}$ is determined by $\rho^{\prime}\left(z_{m}\right) \rightarrow \infty$ and is given by

$$
z_{m}=R-\frac{R^{3}}{6}\left(2+\frac{\beta_{3}^{2}}{16}\right)(5-8 \log 2) \mu_{0}^{2}+\mathcal{O}\left(\mu_{0}^{4}\right) .
$$

Using these results in (4.19) and introducing a cut-off $z=\epsilon$, we can calculate the HEE up to quadratic in $\mu_{0}$

$$
S_{A}^{\mathrm{reg}}=\frac{2 \pi L^{2}}{4 G_{4}} \int_{\epsilon}^{z_{m}} d z \mathcal{L}_{A}=\frac{\pi L^{2}}{2 G_{4}}\left(\frac{R}{\epsilon}-1-\frac{32+\beta_{3}^{2}}{24} R^{2} \mu_{0}^{2}+\cdots\right),
$$

where here and in the following equations, the ellipses denote terms which are higher order in $\mu_{0}$. Since we are considering the $\alpha=2$ case, only the first counter term in $(2.20)$ is required to cancel the divergences. The induced metric on the boundary curve $\partial \Sigma_{A}^{\epsilon}$ is

$$
\tilde{\gamma}_{\theta \theta}=L^{2} \frac{1+h(\epsilon)}{\epsilon^{2}} \rho(\epsilon)^{2} .
$$

Then counter term is

$$
S_{\mathrm{ct}}^{(1)}=-\frac{L}{4 G_{4}} \int d \theta \sqrt{\tilde{\gamma}_{\theta \theta}}=-\frac{\pi L^{2}}{2 G_{4}} \frac{R}{\epsilon}+\mathcal{O}\left(\epsilon^{\alpha}\right) .
$$

From the renormalized HEE for $\alpha>1$, which was constructed in (2.21), we obtain

$$
S_{A}^{\mathrm{ren}}=-\frac{\pi L^{2}}{2 G_{4}}\left(1+\frac{32+\beta_{3}^{2}}{24} R^{2} \mu_{0}^{2}+\cdots\right) .
$$

Here the negative sign of the contribution from the mass deformation (a relevant perturbation) is related to the $F$-theorem in the 3 -dimensional CFT. 
Similarly, the HSC, which is identified with the volume $\mathcal{B}_{\epsilon}$ enclosed by the disc $A$ and the static minimal area surface $\Sigma_{A}^{\epsilon}$ at a constant time slice $t=t_{0}$, is given by

$$
\mathcal{C}_{A}^{\mathrm{reg}}=\frac{V\left(\mathcal{B}_{\epsilon}\right)}{8 \pi L G_{4}}
$$

See figure 1. Using the bulk coordinates $x^{m}=(z, \rho, \theta)$ on the constant time slice, the volume is given by

$$
\begin{aligned}
\mathcal{C}_{A}^{\mathrm{reg}} & =\frac{L^{2}}{8 \pi G_{4}} \int_{0}^{2 \pi} d \theta \int_{\epsilon}^{z_{m}} d z \int_{0}^{\rho(z)} d \rho \frac{\rho}{z^{3}}[1+h(z)] \\
& =\frac{\pi L^{2}}{16 \pi G_{4}}\left[-1+\frac{R^{2}}{\epsilon^{2}}+2 \log \left(\frac{\epsilon}{R}\right)+\left(\frac{32+\beta_{3}^{2}}{8}\right)\left(1+\log \left(\frac{\epsilon}{R}\right)\right) R^{2} \mu_{0}^{2}+\cdots\right] .
\end{aligned}
$$

The above result contains three divergent terms. Those divergences are regulated by the three counter terms that were introduced in the subsection 3.1. The first counter term is given by

$$
\begin{aligned}
\mathcal{C}_{\mathrm{ct}}^{(1)} & =-\frac{1}{16 \pi G_{4}} \int_{0}^{2 \pi} d \theta \int_{0}^{\rho(\epsilon)} d \rho \sqrt{\operatorname{det} h_{i j}}=-\frac{L^{2}}{8 G_{4}} \frac{1+h(\epsilon)}{\epsilon^{2}} \int_{0}^{\rho(\epsilon)} \rho d \rho \\
& =\frac{L^{2}}{16 G_{4}}\left[-\frac{R^{2}}{\epsilon^{2}}+1+\left(\frac{32+\beta_{3}^{2}}{16}\right) R^{2} \mu_{0}^{3}+\cdots\right] .
\end{aligned}
$$

Recalling that in the case we are considering here, $\alpha=2$, the second counter term is given by

$$
\begin{aligned}
\mathcal{C}_{\mathrm{ct}}^{(2)} & =-\frac{1}{64 \pi G_{4}} \ln \left(\frac{\epsilon}{R}\right) \int_{0}^{2 \pi} d \theta \int_{0}^{\rho(\epsilon)} d \rho \sqrt{\operatorname{det} h_{i j}}\left(T^{2}+\Psi^{2}\right)=\frac{\pi L^{2}}{8 \pi G_{4}} \ln \left(\frac{\epsilon}{R}\right) \int_{0}^{\rho(\epsilon)} d \rho \frac{\rho}{\epsilon} h^{\prime}(\epsilon) \\
& =-\frac{L^{2}}{16 G_{4}}\left[\ln \left(\frac{\epsilon}{R}\right)\left(\frac{32+\beta_{3}^{2}}{8}\right) R^{2} \mu_{0}^{2}+\cdots\right] .
\end{aligned}
$$

In order to calculate the third counter term, we need to obtain the extrinsic curvature of the boundary curve $\partial \Sigma_{A}^{\epsilon}$ using the embedding $x^{i}=(\rho=\rho(\epsilon), \theta)$. The induced metric and the extrinsic curvature are given by

$$
\begin{array}{rlrl}
\tilde{\gamma}_{\theta \theta}=L^{2} \frac{1+h(\epsilon)}{\epsilon^{2}} \rho(\epsilon)^{2}, & \tilde{K}_{\rho \rho}=0, & \tilde{K}_{\rho \theta}=\tilde{K}_{\theta \rho}=0, \\
\tilde{K}_{\theta \theta}=-\frac{L \rho(\epsilon)}{\epsilon} \sqrt{1+h(\epsilon)}, & \tilde{K}=g^{i j} \tilde{K}_{i j}=-\left(\frac{L \rho(\epsilon)}{\epsilon} \sqrt{1+h(\epsilon)}\right)^{-1} .
\end{array}
$$

Therefore, the third counter term is obtained as

$$
\tilde{\mathcal{C}}_{\mathrm{ct}}=\frac{L^{2}}{16 \pi G_{4}} \ln \left(\frac{\epsilon}{R}\right) \int_{0}^{2 \pi} d \theta \sqrt{\operatorname{det} \tilde{\gamma} \theta \theta} \tilde{K}=-\frac{L^{2}}{8 G_{4}} \ln \left(\frac{\epsilon}{R}\right) .
$$

Note that, unlike the first two counter terms, this one is exact and has no $\mu_{0}$ corrections, which means that it is independent of the scalar deformation. This is because, when we deform the pure $\mathrm{AdS}_{4}$ space to obtain an asymptotically $\mathrm{AdS}_{4}$ space by adding scalar deformations, the induced metric on the cut-off surface $\partial \mathcal{M}_{\epsilon}$ as well as those on the minimal 
surface $\Sigma_{A}$ get deformed, however, the boundary curve $\partial \Sigma_{A}$ of the minimal surface remains the same. Since the third counter term is built by the invariants on this codimension-three boundary curve, it is independent of the scalar deformation. In general, as we have shown by using the examples of $d=3$ and $d=4$ in the previous section, there are always divergent terms which are independent of the scalar deformation, it was logarithmic in $d=3$ and $\mathcal{O}\left(\epsilon^{-1}\right)$ in $d=4$. The counter terms which cancels these divergence are always built from the invariants on the codimension-three boundary of the minimal hyper-surface $\Sigma_{A}$. Therefore, the general claim in the literature, which states that the counter terms to cancel all the divergences encountered in the complexity calculations are built from the invariants on the codimension-two cut-off hyper-surface $\partial \mathcal{M}_{\epsilon}$, does not account for those divergences.

Finally, adding the three counter terms to the regularized HSC in (4.29) using the renormalized HSC constructed in (3.13) and (3.14), we obtain

$$
\mathcal{C}_{A}^{\text {ren }}=\frac{3 L^{2}}{16^{2} G_{4}}\left(\left(32+\beta_{3}^{2}\right) R^{2} \mu_{0}^{2}+\cdots\right),
$$

where we notice that the renormalized HSC is vanishing in the absence of the relevant perturbation. Therefore, the contribution for the HSC by the mass deformation (a relevant deformation) is always positive, while that for the HEE is negative. From the renormalized HEE in (4.27), we obtain the relation for the disk entangling region up to $\mu_{0}^{2}$-order,

$$
\Delta \mathcal{C}_{A}^{\mathrm{ren}}=-\frac{9}{16 \pi^{2}} \Delta S_{A}^{\mathrm{ren}}
$$

where $\Delta S_{A}^{\text {ren }}$ and $\Delta \mathcal{C}_{A}^{\text {ren }}$, respectively, are the variations of the renormalized HEE and HSC due to the relevant perturbations. The properties of the relation (4.35) were investigated in [55]. However, in our case, it is not clear that the relation (4.35) is satisfied for higher $\mu_{0}$-orders and different shapes of subregions.

\section{Conclusion}

In this paper, we examined the renormalization of HEE and $\mathrm{HSC}$ of general entangling subregions on the asymptotically $\mathrm{AdS}_{4}$ and $\mathrm{AdS}_{5}$ geometries under relevant perturbations originated from a bulk scalar field. We considered the HSC of the CV conjecture and omitted the case of the asymptotically $\mathrm{AdS}_{3}$ geometry, which is similar to the case of the $\mathrm{AdS}_{5}$ geometry, whereas we have obtained the counter terms that cancel few leading divergences in case of asymptotically $\mathrm{AdS}_{d+1}$ geometry when $d>4$. In order to renormalize these quantities in a coordinate independent way, we explicitly constructed universal counter terms using the holographic renormalization method.

For the divergences of the HEE on an asymptotically $\mathrm{AdS}_{d+1}$ geometry, the proposed counter terms are integrals of the curvature invariants on the $(d-2)$-dimensional boundary of the RT minimal hyper-surface. We pointed out that curvature invariants on the boundary of the RT minimal hyper-surface are independent of the bulk stress tensor. On the other hand, the HEE contains subleading divergences whose coefficients are determined by the back reaction of the stress tensor on the geometry. We showed that the counter terms that cancel these subleading divergence, must contain invariants of the bulk matter fields 
in addition to the curvature invariants on the boundary of the RT hyper-surface. We have determined the exact forms of these counter terms in the asymptotically $\mathrm{AdS}_{4}$ and $\mathrm{AdS}_{5}$ geometries with arbitrary shapes of entangling regions.

Taking lesson from the renormalization of the HEE, the counter terms for the divergences in HSC were proposed as integrals of the curvature invariants on the $(d-1)$ dimensional cut-off hyper-surface at $z=\epsilon$, with $z$ being the holographic coordinate. In this case the curvature invariants on the cut-off hyper-surface are dependent on the bulk stress tensor. Therefore, it looks natural to build the counter terms just from the integrals of the curvature invariants on the $(d-1)$-dimensional cut-off boundary. However, we pointed out that there are always divergences that are independent of the scalar deformations and can not be cancelled by the invariants on the $(d-1)$-dimensional cut-off boundary. The counter terms for those divergences are expressed in terms of integrals of curvature invariants on the boundary of the RT minimal hyper-surface. We argued that the existence of these divergence is attributed to the fact that the $(d-1)$-dimensional cut-off boundary meets the $(d-2)$-dimensional boundary of the RT hyper-surface and gets the UV divergence. We showed that the complete counter terms for the divergences of HSC are expressed as integrals of the curvature invariants on the $(d-1)$-dimensional cut-off boundary plus integrals of the curvature invariants on the $(d-2)$-dimensional boundary of the RT hyper-surface.

We have tested our general construction of the renormalized HEE and HSC for an asymptotically $\mathrm{AdS}_{4}$ geometry, which was obtained from the non-linear KK reduction of the 11-dimensional LLM geometry. We obtained coordinate independent finite results for both HEE and HSC with a disk shape of entangling region. For our convenience of the coordinate choice for the disk, we used a different mapping with (2.8). However, the counter terms for the HEE and HSC have the same form with those in subsections 2.1 and 3.1, respectively.

Intriguingly, we found that the coordinate independent renormalization of the HSC in the asymptotically $\mathrm{AdS}_{5}$ is not possible in the range $0<\alpha<1$, i.e., $0<\Delta<\frac{1}{2}$ and $\frac{7}{2}<$ $\Delta<4$ of the relevant operators in the 4-dimensional dual field theory. That is, a divergent term in that range of $\alpha$ cannot be cancelled out by adding any curvature invariant. We also noticed that the case of $\frac{7}{2}<\Delta<4$ does not violate the unitary bound $(\Delta \geq 1)$ for primary operators. Therefore, the problem of the non-renormalizability of the HSC in the asymptotically $\mathrm{AdS}_{5}$ is genuine in this range of $\alpha$. It will be interesting if one figures out the physical reason of this phenomenon by investigating cases of other dimensions and other HSC conjectures, for instance, the HSC in the CA conjecture.

\section{Acknowledgments}

We would like to thank Kyung Kiu Kim and Chanyong Park for useful comments and discussions. This work was supported by the National Research Foundation of Korea (NRF) grant with grant number NRF-2018R1D1A1B07048061 (D.J.), NRF2019R1F1A1056815 (Y.K.), NRF-2020R1A2C1014371, NRF-2019R1F1A1059220, NRF2019R1A6A1A10073079 (O.K.), and NRF-2017R1D1A1B03032523 (D.T.). Y.K. and O.K. were also supported by NRF Bilateral Joint Research Projects (NRF-JSPS collaboration) "String Axion Cosmology". 


\section{A Renormalized HEE in pure $\mathrm{AdS}_{d+1}$ geometry}

In order to demonstrate the complete renoramilazation of HEE in arbitrary dimensions, lets consider the simple case of an $\mathrm{AdS}_{d+1}$ geometry with the entangling surface $A$ is a disc. The metric in the Fefferman-Graham coordinates is given by

$$
d s^{2}=\frac{L^{2}}{z^{2}}\left(d z^{2}-d t^{2}+d \rho^{2}+\rho^{2} d \Omega_{d-2}^{2}\right),
$$

where $d \Omega_{d-2}^{2}$ is the metric on $S^{d-2}$, with coordinates $x^{a}=\left\{\theta_{1}, \cdots, \theta_{d-2}\right\}$. The RT hypersurface $\Sigma_{A}$ is a $(d-1)$-dimensional manifold parametrized by the embedding

$$
x^{p}=\left(z, t=t_{0}, \rho=\rho(z), \theta_{1}, \cdots, \theta_{d-2},\right) .
$$

Introducing the coordinates on the minimal surface as $\sigma^{\alpha}=\left(\rho, \theta_{1}, \cdots, \theta_{d-2}\right)$, the induce metric is given by

$$
\gamma_{\rho \rho}=\frac{L^{2}}{z^{2}}\left(1+\frac{1}{\rho^{\prime}(z)^{2}}\right), \quad \gamma_{\rho a}=0, \quad \gamma_{a b}=\frac{L^{2}}{z^{2}} \rho(z)^{2} \tilde{g}_{a b},
$$

where $\tilde{g}_{a b}$ is the metric elements of the $S^{d-2}$. The HEE which is determined by the area of the RT hyper-surface is

$$
S_{A}=\frac{L^{d-1} \Omega_{d-2}}{4 G_{n+1}} \int_{0}^{z_{m}} d z \frac{\rho(z)^{d-2}}{z^{d-1}} \sqrt{1+\rho^{\prime}(z)^{2}},
$$

where $\Omega_{d-2}=2 \frac{\pi^{\frac{d-1}{2}}}{\Gamma\left(\frac{d-1}{2}\right)}$ is the volume of the $(d-2)$-sphere. The $\rho(z)$ is determined by solving the Euler-Lagrangian equation from this action, which is

$$
z \rho(z) \rho^{\prime \prime}(z)-\left(\rho^{\prime}(z)^{2}+1\right)\left((d-1) \rho(z) \rho^{\prime}(z)+(d-2) z\right)=0 .
$$

An exact solution of this equation can be obtained, unlike the case of the asymptotically AdS geometry or arbitrary entangling surface. With the boundary condition $\rho(z=0)=R$ the exact solution is

$$
\rho(z)=\sqrt{R^{2}-z^{2}}
$$

Plugging this exact solution into (A.4), expanding in powers of $z$, and introducing the cut-off $z=\epsilon$, the regularized HEE is given by

$$
S_{A}^{\mathrm{reg}}=\frac{L^{d-1} R^{d-2}}{4 G_{d+1}} \Omega_{d-2} \int_{\epsilon}^{z_{m}} d z\left[\frac{1}{z^{d-1}}+\sum_{n=1}^{\infty} \frac{(-1)^{n}}{2^{n} n ! R^{2 n}} z^{2 n-d+1} \prod_{m=1}^{n}(d-2 m-1)\right] .
$$

To evaluating the $z$ integration, lets assume $d>2$ (where there is only the leading order logarithmic divergence) and also consider the odd and even $d$ cases separately. In the case of odd $d$, the least divergent term is $\mathcal{O}\left(\epsilon^{-1}\right)$ and the result of the integration is

$$
S_{A}^{\mathrm{reg}}=\frac{L^{d-1} R^{d-2}}{4 G_{d+1}} \Omega_{d-2}\left[\frac{1}{(d-2) \epsilon^{d-2}}+\sum_{n=1}^{\frac{d-3}{2}} \frac{(-1)^{n}}{2^{n} n ! R^{2 n}} \frac{\epsilon^{2 n-d+2}}{(d-2-2 n)} \prod_{m=1}^{n}(d-2 m-1)+\cdots\right],
$$


whereas in the case of even $d$, the least divergent term is logarithmic, and we can write

$$
\begin{aligned}
S_{A}^{\mathrm{reg}}= & \frac{L^{d-1} R^{d-2}}{4 G_{d+1}} \Omega_{d-2}\left[\frac{1}{(d-2) \epsilon^{d-2}}+\sum_{n=1}^{\frac{d-4}{2}} \frac{(-1)^{n}}{2^{n} n ! R^{2 n}} \frac{\epsilon^{2 n-d+2}}{(d-2-2 n)} \prod_{m=1}^{n}(d-2 m-1)+\cdots\right] \\
& -\frac{L^{d-1}}{4 G_{d+1}} \Omega_{d-2}\left[\frac{(-1)^{\frac{d-2}{2}}}{2^{\frac{d-2}{2}}\left(\frac{d-2}{2}\right) !} \log \left(\frac{\epsilon}{R}\right) \prod_{m=1}^{\frac{d-2}{2}}(d-2 m-1)+\cdots\right] .
\end{aligned}
$$

Here the ellipses denote only finite terms, which means that we have explicitly expressed all divergent terms. This is difficult to do in a more general set up because solving the notoriously complex minimal area equations in (2.49) beyond few orders in $z$ is very challenging.

The counter terms to cancel these divergences are composed of the invariants of the induced metric and the extrinsic curvature on the boundary space $\partial \Sigma_{A}^{\epsilon}$. Using the embedding $x^{i}=\left(\rho=\rho(\epsilon), \theta_{1}, \cdots, \theta_{d-2}\right)$, the induced metric and the extrinsic curvature are given by

$$
\begin{array}{ll}
\tilde{\gamma}_{a b}=\frac{L^{2}}{\epsilon^{2}} \rho(\epsilon)^{2} \tilde{g}_{a b}, & \tilde{K}_{\rho \rho}=0, \quad \tilde{K}_{\rho a}=\tilde{K}_{a \rho}=0, \\
\tilde{K}_{a b}=-\frac{L}{\epsilon} \rho(\epsilon) \tilde{g}_{a b}, & \tilde{K}=g^{i j} \tilde{K}_{i j}=-(d-2) \frac{\epsilon}{L \rho(\epsilon)} .
\end{array}
$$

We note that other invariants of the extrinsic curvature can be written as power of $\tilde{K}$. For, instance

$$
\tilde{K}^{i j} \tilde{K}_{i j}=\frac{1}{d-2} \tilde{K}^{2}, \quad \tilde{K}^{i j} \tilde{K}_{j m} \tilde{K}_{i}^{m}=\frac{1}{(d-2)^{2}} \tilde{K}^{3}, \quad \cdots
$$

Therefore, recalling also that the intrinsic curvature $\tilde{R}_{a b}$ is vanishing, the counter terms to remove the divergences in (A.8) and (A.9) are composed of only the trace of the extrinsic curvature.

Starting with the leading order divergence and going order by order, it is not difficult to find the pattern of the necessary counter terms to cancel the $\mathcal{O}\left(\epsilon^{2(k+1)-d}\right)$ divergence with $k=0,1,2, \cdots$. It is given by

$$
S_{\mathrm{ct}}^{(k+1)}=\frac{(-1)^{k+1}(2 k-1) ! ! L^{2 k+1}}{4 G_{d+1} 2^{k} k !(d-2 k-2)(d-2)^{2 k}} \int d^{d-2} \theta \sqrt{\tilde{\gamma}_{a b}} \tilde{K}^{2 k},
$$

where $(2 k-1) ! !=(2 k-1)(2 k-3)(2 k-5) \cdots 1$ and $(-1) ! !=1$. Adding all the counter terms up to this order to the regularized HEE in (A.8) and (A.9), we obtain

$$
\begin{aligned}
S_{A}^{\mathrm{reg}}+S_{\mathrm{ct}}^{(1)}+S_{\mathrm{ct}}^{(2)}+\cdots+S_{\mathrm{ct}}^{(k+1)} & \frac{L^{d-1} R^{d-2} \Omega_{d-2}}{4 G_{d+1}}\left[\sum _ { n = k + 1 } ^ { \frac { d - 3 } { 2 } } \frac { ( - 1 ) ^ { n } \epsilon ^ { 2 n - d + 2 } } { 2 ^ { n } n ! R ^ { 2 n } } \left(\frac{\prod_{m=1}^{n}(d-2 m-1)}{d-2-2 n}\right.\right. \\
& \left.\left.-\left((2 k-1) ! !\left(\begin{array}{c}
n \\
k
\end{array}\right) \prod_{m=k+2}^{n}+(2 k-3) ! !\left(\begin{array}{c}
n \\
k-1
\end{array}\right) \prod_{k+1}^{n}+\cdots+\left(\begin{array}{c}
n \\
0
\end{array}\right) \prod_{m=2}^{n}\right)(d-2 m)\right)+\cdots\right],
\end{aligned}
$$


when $d$ is odd and

$$
\begin{aligned}
& S_{A}^{\mathrm{reg}}+S_{\mathrm{ct}}^{(1)}+S_{\mathrm{ct}}^{(2)}+\cdots+S_{\mathrm{ct}}^{(k+1)} \\
& =\frac{L^{d-1} R^{d-2} \Omega_{d-2}}{4 G_{d+1}}\left[\sum _ { n = k + 1 } ^ { \frac { d - 4 } { 2 } } \frac { ( - 1 ) ^ { n } \epsilon ^ { 2 n - d + 2 } } { 2 ^ { n } n ! R ^ { 2 n } } \left(\frac{\prod_{m=1}^{n}(d-2 m-1)}{d-2-2 n}\right.\right. \\
& \left.\left.-\left((2 k-1) ! !\left(\begin{array}{l}
n \\
k
\end{array}\right) \prod_{m=k+2}^{n}+(2 k-3) ! !\left(\begin{array}{c}
n \\
k-1
\end{array}\right) \prod_{m=k+1}^{n}+\cdots+\left(\begin{array}{c}
n \\
0
\end{array}\right) \prod_{m=2}^{n}\right)(d-2 m)\right)+\cdots\right] \\
& -\frac{L^{d-1}}{4 G_{d+1}} \Omega_{d-2}\left[\frac{(-1)^{\frac{d-2}{2}}}{2^{\frac{d-2}{2}}\left(\frac{d-2}{2}\right) !} \log \left(\frac{\epsilon}{R}\right) \prod_{m=1}^{\frac{d-2}{2}}(d-2 m-1)+\cdots\right],
\end{aligned}
$$

when $d$ is even. For odd $d$, the least divergent term is at order of $k=\frac{d-3}{2}$ and the corresponding counter term is

$$
S_{\mathrm{ct}}^{\left(\frac{d-1}{2}\right)}=\frac{(-1)^{\frac{d-1}{2}}(d-4) ! ! L^{d-2}}{4 G_{d+1} 2^{\frac{d-3}{2}}\left(\frac{d-3}{2}\right) !(d-2)^{d-3}} \int d^{d-2} \theta \sqrt{\tilde{\gamma}_{a b}} \tilde{K}^{d-3},
$$

whereas for even $d$ it is the logarithm divergence and the counter term is

$$
S_{\mathrm{ct}}^{\left(\frac{d}{2}\right)}=-\frac{(-1)^{\frac{d}{2}}(d-3) ! ! L^{d-1}}{4 G_{d+1} 2^{\frac{d-2}{2}}\left(\frac{d-2}{2}\right) !(d-2)^{d-2}} \log \left(\frac{\epsilon}{R}\right) \int d^{d-2} \theta \sqrt{\tilde{\gamma}_{a b}} \tilde{K}^{d-2} .
$$

Therefore, the renormalized HEE is

$$
S_{A}^{\mathrm{ren}}=\left\{\begin{array}{ll}
S_{A}^{\mathrm{reg}}+S_{\mathrm{ct}}^{(1)}+S_{\mathrm{ct}}^{(2)}+\cdots+S_{\mathrm{ct}}^{(k+1)}+\cdots+S_{\mathrm{ct}}^{\left(\frac{d-1}{2}\right)}, & \text { for odd } d, \\
S_{A}^{\mathrm{reg}}+S_{\mathrm{ct}}^{(1)}+S_{\mathrm{ct}}^{(2)}+\cdots+S_{\mathrm{ct}}^{(k+1)}+\cdots+S_{\mathrm{ct}}^{\left(\frac{d}{2}\right)}, & \text { for even } d
\end{array} .\right.
$$

\section{B Renormalized HSC in pure $\operatorname{AdS}_{d+1}$ geometry}

Using the results we have collated in appendix A, the regularized HSC for $\mathrm{AdS}_{d+1}$ geometry and the disc entangling space is given by

$$
\begin{aligned}
\mathcal{C}_{A}^{\text {reg }} & =\frac{L^{d-1}}{8 \pi G_{d+1}} \int d^{d-2} \theta \sqrt{\tilde{g}_{a b}} \int_{\epsilon}^{z_{m}} d z \int_{0}^{\rho(z)} d \rho \frac{\rho^{d-2}}{z^{d}} \\
& =\frac{L^{d-1} \Omega_{d-2} R^{d-1}}{8 \pi(d-1) G_{d+1}} \int_{\epsilon}^{z_{m}} d z\left[\sum_{n=0}^{\infty} \frac{(-1)^{n} z^{2 n-d}}{2^{n} n ! R^{2 n}} \prod_{m=1}^{n}(d-2 m+1)\right] .
\end{aligned}
$$

As in the case of HEE, the integration over the $z$ coordinate gives

$$
\mathcal{C}_{A}^{\mathrm{reg}}=\frac{L^{d-1} \Omega_{d-2} R^{d-1}}{8 \pi(d-1) G_{d+1}}\left[\sum_{n=0}^{\frac{d-2}{2}} \frac{(-1)^{n} \epsilon^{2 n-d+1}}{2^{n} n ! R^{2 n}(d-2 n-1)} \prod_{m=1}^{n}(d-2 m+1)+\cdots\right],
$$


for even $d$ and for odd $d$ it is

$$
\begin{aligned}
\mathcal{C}_{A}^{\mathrm{reg}}= & \frac{L^{d-1} \Omega_{d-2} R^{d-1}}{8 \pi(d-1) G_{d+1}}\left[\sum_{n=0}^{\frac{d-3}{2}} \frac{(-1)^{n} \epsilon^{2 n-d+1}}{2^{n} n ! R^{2 n}(d-2 n-1)} \prod_{m=1}^{n}(d-2 m+1)+\cdots\right] \\
& -\frac{L^{d-1} \Omega_{d-2}}{8 \pi(d-1) G_{d+1}}\left[\frac{(-1)^{\frac{d-1}{2}}}{2^{\frac{d-1}{2}} \frac{d-1}{2} !} \log \left(\frac{\epsilon}{R}\right) \prod_{m=1}^{\frac{d-1}{2}}(d-2 m+1)+\cdots\right] .
\end{aligned}
$$

As we have discussed in section 3 , in the absence of the matter deformation, the counter terms that cancel the divergences in (B.2) and (B.3) are composed of the invariants of the intrinsic and extrinsic curvatures on both the $(d-1)$-dimensional cut-off space $\partial \mathcal{M}_{\epsilon}$ and the $(d-2)$-dimensional boundary $\partial \Sigma_{A}^{\epsilon}$ of the RT minimal area hyper-surface. The induced metric $h_{i j}$ and the extrinsic curvature $K_{i j}$ on the cut-off surface $\partial \mathcal{M}_{\epsilon}$ are given by

$$
h_{\rho \rho}=\frac{L^{2}}{\epsilon^{2}}, \quad h_{a b}=\frac{L^{2} \rho(\epsilon)^{2}}{\epsilon^{2}} \tilde{g}_{a b}, \quad K_{\rho \rho}=-\frac{L}{\epsilon^{2}}, \quad K_{a b}=-\frac{L \rho(\epsilon)^{2}}{\epsilon^{2}} \tilde{g}_{a b},
$$

whereas those of $\partial \Sigma_{A}^{\epsilon}$ are as in (A.10).

As always the counter term that cancels the leading order divergence is proportional to the volume of the $(d-1)$-dimensional cut-off space $\operatorname{Vol}\left(\partial \mathcal{M}_{\epsilon}\right)$ and it is given by

$$
\mathcal{C}_{\mathrm{ct}}^{(1)}=-\frac{1}{8 \pi(d-1) G_{d+1}} \int d^{d-2} \theta \int_{0}^{\rho(\epsilon)} d \rho \sqrt{\operatorname{det} h_{i j}}
$$

Since $K=g^{i j} K_{i j}$ in (B.4) is constant and the intrinsic curvature is zero, there is no other counter term obtained from the curvature invariants on the $(d-1)$-dimensional boundary space $\partial \mathcal{M}_{\epsilon}$. Therefore, in order to calculate counter terms for all subleading divergences, we need to use the extrinsic curvature of the boundary curve $\partial \Sigma_{A}^{\epsilon}$ in (A.10). As a result, the counter term that cancels the $\mathcal{O}\left(\epsilon^{2 k-d+3}\right)$ subleading divergence with $k=0,1,2, \cdots$, is

$$
\tilde{\mathcal{C}}_{\mathrm{ct}}^{(k+1)}=\frac{(-1)^{k+1} L^{2 k+2}}{8 \pi(d-1)(d-2)^{2 k+1}(d-2 k-3) G_{d+1}} \int d^{d-2} \theta \sqrt{\operatorname{det} \tilde{\gamma}_{a b}} \tilde{K}^{2 k+1}
$$

Adding these counter terms for the subleading divergences as well as that of the leading divergence in (B.5), the HSC in (B.2) and (B.3) becomes

$$
\begin{aligned}
& \mathcal{C}_{A}^{\mathrm{reg}}+\mathcal{C}_{\mathrm{ct}}^{(1)}+\tilde{\mathcal{C}}_{\mathrm{ct}}^{(1)}+\cdots+\tilde{\mathcal{C}}_{\mathrm{ct}}^{(k+1)} \\
& =\frac{L^{d-1} \Omega_{d-2} R^{d-1}}{8 \pi(d-1) G_{d+1}}\left[\sum _ { n = k + 2 } ^ { \frac { d - 2 } { 2 } } \frac { ( - 1 ) ^ { n } \epsilon ^ { 2 n - d + 1 } } { 2 ^ { n } n ! R ^ { 2 n } } \left(\frac{\prod_{m=1}^{n}}{d-2 n-1}-\left(\begin{array}{c}
n \\
0
\end{array}\right) \prod_{m=2}^{n}-2\left(\begin{array}{c}
n \\
1
\end{array}\right) \prod_{m=3}^{n}\right.\right. \\
& \left.\left.\quad-8\left(\begin{array}{c}
n \\
2
\end{array}\right) \prod_{m=4}^{n}-\cdots-2^{k+1}(k+1) !\left(\begin{array}{c}
n \\
k+1
\end{array}\right) \prod_{m=k+3}^{n}\right)(d-2 m+1)+\cdots\right],
\end{aligned}
$$


for even $d$ whereas for odd $d$ it is

$$
\begin{aligned}
& \mathcal{C}_{A}^{\mathrm{reg}}+\mathcal{C}_{\mathrm{ct}}^{(1)}+\tilde{\mathcal{C}}_{\mathrm{ct}}^{(1)}+\cdots+\tilde{\mathcal{C}}_{\mathrm{ct}}^{(k+1)} \\
& =\frac{L^{d-1} \Omega_{d-2} R^{d-1}}{8 \pi(d-1) G_{d+1}}\left[\sum _ { n = k + 2 } ^ { \frac { d - 3 } { 2 } } \frac { ( - 1 ) ^ { n } \epsilon ^ { 2 n - d + 1 } } { 2 ^ { n } n ! R ^ { 2 n } } \left(\frac{\prod_{m=1}^{n}}{d-2 n-1}-\left(\begin{array}{c}
n \\
0
\end{array}\right) \prod_{m=2}^{n}-2\left(\begin{array}{c}
n \\
1
\end{array}\right) \prod_{m=3}^{n}\right.\right. \\
& \left.\left.\quad-8\left(\begin{array}{c}
n \\
2
\end{array}\right) \prod_{m=4}^{n}-\cdots-2^{k+1}(k+1) !\left(\begin{array}{c}
n \\
k+1
\end{array}\right) \prod_{m=k+3}^{n}\right)(d-2 m+1)+\cdots\right] \\
& -\frac{L^{d-1} \Omega_{d-2}}{8 \pi(d-1) G_{d+1}}\left[\frac{(-1)^{\frac{d-1}{2}}}{2^{\frac{d-1}{2}} \frac{d-1}{2} !} \log \left(\frac{\epsilon}{R}\right) \prod_{m=1}^{\frac{d-1}{2}}(d-2 m+1)+\cdots\right] .
\end{aligned}
$$

Proceeding like this, one can cancel all the divergences up to the last one, which for even $d$ corresponds to $k+1=\frac{d-2}{2}$ and the counter term is

$$
\tilde{\mathcal{C}}_{\mathrm{ct}}^{\left(\frac{d-2}{2}\right)}=\frac{(-1)^{\frac{d-2}{2}} L^{d-2}}{8 \pi(d-1)(d-2)^{d-3} G_{d+1}} \int d^{d-2} \theta \sqrt{\operatorname{det} \tilde{\gamma}_{a b}} \tilde{K}^{d-3},
$$

and for odd $d$ it is the logarithmic divergence with the counter term

$$
\tilde{\mathcal{C}}_{\mathrm{ct}}^{\left(\frac{d-1}{2}\right)}=-\frac{(-1)^{\frac{d-1}{2}} L^{d-1}}{8 \pi(d-1)(d-2)^{d-2} G_{d+1}} \log \left(\frac{\epsilon}{R}\right) \int d^{d-2} \theta \sqrt{\operatorname{det} \tilde{\gamma}_{a b}} \tilde{K}^{d-2} .
$$

Open Access. This article is distributed under the terms of the Creative Commons Attribution License (CC-BY 4.0), which permits any use, distribution and reproduction in any medium, provided the original author(s) and source are credited.

\section{References}

[1] J.M. Maldacena, The Large $N$ limit of superconformal field theories and supergravity, Int. J. Theor. Phys. 38 (1999) 1113 [hep-th/9711200] [INSPIRE].

[2] S.S. Gubser, I.R. Klebanov and A.M. Polyakov, Gauge theory correlators from noncritical string theory, Phys. Lett. B 428 (1998) 105 [hep-th/9802109] [INSPIRE].

[3] E. Witten, Anti-de Sitter space and holography, Adv. Theor. Math. Phys. 2 (1998) 253 [hep-th/9802150] [INSPIRE].

[4] S. Ryu and T. Takayanagi, Holographic derivation of entanglement entropy from AdS/CFT, Phys. Rev. Lett. 96 (2006) 181602 [hep-th/0603001] [INSPIRE].

[5] S. Ryu and T. Takayanagi, Aspects of Holographic Entanglement Entropy, JHEP 08 (2006) 045 [hep-th/0605073] [INSPIRE].

[6] V.E. Hubeny, M. Rangamani and T. Takayanagi, A Covariant holographic entanglement entropy proposal, JHEP 07 (2007) 062 [arXiv:0705.0016] [INSPIRE].

[7] D. Stanford and L. Susskind, Complexity and Shock Wave Geometries, Phys. Rev. D 90 (2014) 126007 [arXiv:1406.2678] [INSPIRE]. 
[8] A.R. Brown, D.A. Roberts, L. Susskind, B. Swingle and Y. Zhao, Complexity, action, and black holes, Phys. Rev. D 93 (2016) 086006 [arXiv: 1512.04993] [InSPIRE].

[9] A.R. Brown, D.A. Roberts, L. Susskind, B. Swingle and Y. Zhao, Holographic Complexity Equals Bulk Action?, Phys. Rev. Lett. 116 (2016) 191301 [arXiv:1509.07876] [INSPIRE].

[10] M. Alishahiha, Holographic Complexity, Phys. Rev. D 92 (2015) 126009 [arXiv:1509.06614] [INSPIRE].

[11] O. Ben-Ami and D. Carmi, On Volumes of Subregions in Holography and Complexity, JHEP 11 (2016) 129 [arXiv: 1609.02514] [INSPIRE].

[12] D. Carmi, R.C. Myers and P. Rath, Comments on Holographic Complexity, JHEP 03 (2017) 118 [arXiv: 1612.00433] [INSPIRE].

[13] P. Roy and T. Sarkar, Note on subregion holographic complexity, Phys. Rev. D 96 (2017) 026022 [arXiv: 1701.05489] [INSPIRE].

[14] E. Bakhshaei, A. Mollabashi and A. Shirzad, Holographic Subregion Complexity for Singular Surfaces, Eur. Phys. J. C 77 (2017) 665 [arXiv: 1703.03469] [InSPIRE].

[15] P. Roy and T. Sarkar, Subregion holographic complexity and renormalization group flows, Phys. Rev. D 97 (2018) 086018 [arXiv:1708.05313] [INSPIRE].

[16] R. Abt et al., Topological Complexity in AdS $S_{3} / C F T_{2}$, Fortsch. Phys. 66 (2018) 1800034 [arXiv: 1710.01327] [INSPIRE].

[17] B. Chen, W.-M. Li, R.-Q. Yang, C.-Y. Zhang and S.-J. Zhang, Holographic subregion complexity under a thermal quench, JHEP 07 (2018) 034 [arXiv: 1803.06680] [INSPIRE].

[18] C.A. Agón, M. Headrick and B. Swingle, Subsystem Complexity and Holography, JHEP 02 (2019) 145 [arXiv: 1804.01561] [INSPIRE].

[19] R. Abt, J. Erdmenger, M. Gerbershagen, C.M. Melby-Thompson and C. Northe, Holographic Subregion Complexity from Kinematic Space, JHEP 01 (2019) 012 [arXiv:1805.10298] [INSPIRE].

[20] M. Alishahiha, K. Babaei Velni and M.R. Mohammadi Mozaffar, Black hole subregion action and complexity, Phys. Rev. D 99 (2019) 126016 [arXiv:1809.06031] [InSPIRE].

[21] S. Karar, R. Mishra and S. Gangopadhyay, Holographic complexity of boosted black brane and Fisher information, Phys. Rev. D 100 (2019) 026006 [arXiv:1904.13090] [INSPIRE].

[22] A. Bhattacharya, K.T. Grosvenor and S. Roy, Entanglement Entropy and Subregion Complexity in Thermal Perturbations around Pure-AdS Spacetime, Phys. Rev. D 100 (2019) 126004 [arXiv: 1905.02220] [INSPIRE].

[23] R. Auzzi, S. Baiguera, A. Mitra, G. Nardelli and N. Zenoni, Subsystem complexity in warped AdS, JHEP 09 (2019) 114 [arXiv: 1906.09345] [INSPIRE].

[24] A. Ghosh and R. Mishra, Inhomogeneous Jacobi equation and Holographic subregion complexity, arXiv: 1907.11757 [INSPIRE].

[25] R. Auzzi, G. Nardelli, F.I. Schaposnik Massolo, G. Tallarita and N. Zenoni, On volume subregion complexity in Vaidya spacetime, JHEP 11 (2019) 098 [arXiv:1908.10832] [INSPIRE].

[26] E. Caceres, S. Chapman, J.D. Couch, J.P. Hernández, R.C. Myers and S.-M. Ruan, Complexity of Mixed States in QFT and Holography, JHEP 03 (2020) 012 [arXiv: 1909.10557] [INSPIRE]. 


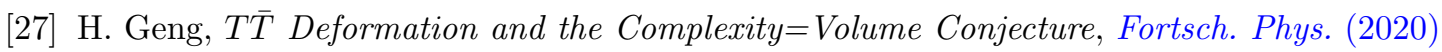
2000036 [arXiv: 1910.08082] [inSPIRE].

[28] M. Miyaji, T. Numasawa, N. Shiba, T. Takayanagi and K. Watanabe, Distance between Quantum States and Gauge-Gravity Duality, Phys. Rev. Lett. 115 (2015) 261602 [arXiv: 1507.07555] [INSPIRE].

[29] H. Liu and M. Mezei, A Refinement of entanglement entropy and the number of degrees of freedom, JHEP 04 (2013) 162 [arXiv:1202.2070] [INSPIRE].

[30] T. Nishioka, Entanglement entropy: holography and renormalization group, Rev. Mod. Phys. 90 (2018) 035007 [arXiv: 1801.10352] [INSPIRE].

[31] R. Emparan, C.V. Johnson and R.C. Myers, Surface terms as counterterms in the AdS/CFT correspondence, Phys. Rev. D 60 (1999) 104001 [hep-th/9903238] [INSPIRE].

[32] S. de Haro, S.N. Solodukhin and K. Skenderis, Holographic reconstruction of space-time and renormalization in the AdS/CFT correspondence, Commun. Math. Phys. 217 (2001) 595 [hep-th/0002230] [INSPIRE].

[33] K. Skenderis, Lecture notes on holographic renormalization, hep-th/0209067 [INSPIRE].

[34] M. Bianchi, D.Z. Freedman and K. Skenderis, Holographic renormalization, Nucl. Phys. B 631 (2002) 159 [hep-th/0112119] [INSPIRE].

[35] M. Taylor and W. Woodhead, Renormalized entanglement entropy, JHEP 08 (2016) 165 [arXiv:1604.06808] [INSPIRE].

[36] G. Anastasiou, I.J. Araya and R. Olea, Topological terms, $A d S_{2 n}$ gravity and renormalized Entanglement Entropy of holographic CFTs, Phys. Rev. D 97 (2018) 106015 [arXiv: 1803.04990] [INSPIRE].

[37] G. Anastasiou, I.J. Araya, A. Guijosa and R. Olea, Renormalized AdS gravity and holographic entanglement entropy of even-dimensional CFTs, JHEP 10 (2019) 221 [arXiv: 1908.11447] [INSPIRE].

[38] R.-Q. Yang, C. Niu and K.-Y. Kim, Surface Counterterms and Regularized Holographic Complexity, JHEP 09 (2017) 042 [arXiv:1701.03706] [INSPIRE].

[39] H. Lin, O. Lunin and J.M. Maldacena, Bubbling AdS space and 1/2 BPS geometries, JHEP 10 (2004) 025 [hep-th/0409174] [INSPIRE].

[40] L.-Y. Hung, R.C. Myers and M. Smolkin, Some Calculable Contributions to Holographic Entanglement Entropy, JHEP 08 (2011) 039 [arXiv:1105.6055] [INSPIRE].

[41] K. Skenderis and M. Taylor, Kaluza-Klein holography, JHEP 05 (2006) 057 [hep-th/0603016] [INSPIRE].

[42] K. Skenderis and M. Taylor, Holographic Coulomb branch vevs, JHEP 08 (2006) 001 [hep-th/0604169] [INSPIRE].

[43] K. Hosomichi, K.-M. Lee, S. Lee, S. Lee and J. Park, $N=5,6$ Superconformal Chern-Simons Theories and M2-branes on Orbifolds, JHEP 09 (2008) 002 [arXiv:0806.4977] [INSPIRE].

[44] J. Gomis, D. Rodriguez-Gomez, M. Van Raamsdonk and H. Verlinde, A Massive Study of M2-brane Proposals, JHEP 09 (2008) 113 [arXiv:0807.1074] [INSPIRE].

[45] D. Jang, Y. Kim, O.-K. Kwon and D.D. Tolla, Mass-deformed ABJM Theory and LLM Geometries: Exact Holography, JHEP 04 (2017) 104 [arXiv: 1612.05066] [INSPIRE]. 
[46] O.-K. Kwon, D. Jang, Y. Kim and D.D. Tolla, Gravity from Entanglement and RG Flow in a Top-down Approach, JHEP 05 (2018) 009 [arXiv: 1712.09101] [INSPIRE].

[47] D. Jang, Y. Kim, O.-K. Kwon and D.D. Tolla, Holography of Massive M2-brane Theory with Discrete Torsion, Eur. Phys. J. C 80 (2020) 224 [arXiv:1906.06881] [InSPIRE].

[48] O. Aharony, O. Bergman, D.L. Jafferis and J. Maldacena, $N=6$ superconformal Chern-Simons-matter theories, M2-branes and their gravity duals, JHEP 10 (2008) 091 [arXiv:0806.1218] [INSPIRE].

[49] S. Cheon, H.-C. Kim and S. Kim, Holography of mass-deformed M2-branes, arXiv:1101.1101 [INSPIRE].

[50] C. Kim, K.K. Kim and O.-K. Kwon, Holographic Entanglement Entropy of Anisotropic Minimal Surfaces in LLM Geometries, Phys. Lett. B 759 (2016) 395 [arXiv:1605.00849] [INSPIRE].

[51] K.K. Kim, O.-K. Kwon, C. Park and H. Shin, Renormalized Entanglement Entropy Flow in Mass-deformed ABJM Theory, Phys. Rev. D 90 (2014) 046006 [arXiv: 1404.1044] [InSPIRE].

[52] V. Balasubramanian, N. Jokela, A. Pönni and A.V. Ramallo, Information flows in strongly coupled ABJM theory, JHEP 01 (2019) 232 [arXiv:1811.09500] [INSPIRE].

[53] B. Ahn, S. Hyun, K.K. Kim, O.-K. Kwon and S.-A. Park, AdS Q-Soliton and Inhomogeneously mass-deformed ABJM Model, JHEP 02 (2020) 132 [arXiv:1911.05783] [INSPIRE].

[54] R. Auzzi and S. Kumar, Non-Abelian Vortices at Weak and Strong Coupling in Mass Deformed ABJM Theory, JHEP 10 (2009) 071 [arXiv:0906.2366] [INSPIRE].

[55] D. Momeni, M. Faizal and R. Myrzakulov, Holographic Cavalieri Principle as a Universal relation between Holographic Complexity and Holographic Entanglement Entropy, Int. J. Mod. Phys. D 27 (2018) 1850103 [arXiv:1703.01337] [INSPIRE]. 